\title{
Research Paper \\ Effect of Mindfulness Therapy and Aromatherapy Massage on Pain Perception, Quality of Life and Sleep Quality in Older Women With Chronic Pain
}

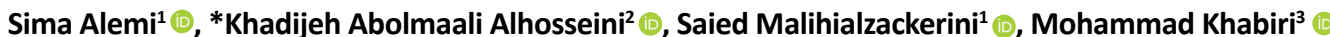

1. Department of Health Psychology, Karaj Branch, Islamic Azad University, Karaj, Iran.

2. Department of Psychology, Faculty of Psychology, Roudehen Branch, Islamic Azad University, Roudehen, Iran.

3. Department of Sport Management, Faculty of Physical Education \& Sport, Tehran University, Tehran, Iran.

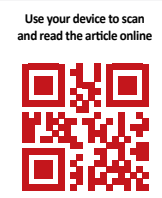

Citation: Alemi S, Abolmaali Alhosseini Kh, Malihialzackerini S, Khabiri M. [Effect of Mindfulness Therapy and Aromatherapy Massage on Pain Perception, Quality of Life and Sleep Quality in Older Women With Chronic Pain (Persian)]. Iranian Journal of Ageing. 2021; 16(2):218-233. https://doi.org/10.32598/sija.16.2.3058.1

Received: 18 Sep 2020

Accepted: $16 \operatorname{Jan} 2021$ Available Online: 01 Jul 2021

Key words: Mindfulness, Aromatherapy, Psychological function, Older women, Chronic pain Quality of life, Pain perception

\section{A B STRACT}

Objectives Aging is one of the phenomena in the field of global health. It is very important to pay attention to older adults psychologically and physically. This study aims to evaluate the effect of mindfulness therapy combined with aromatherapy massage on the psychological functions of older women with chronic pain Methods \& Materials This is a quasi-experimental study with a pre-test, post-test, follow-up design using a control group. The study population consists of all older women with chronic pain referred to hospitals, clinics, and rehabilitation centers in the west of Tehran, Iran in 2017. By using a convenience sampling method, 30 women were selected based on inclusion and exclusion criteria and randomly divided into two matched groups. The intervention group underwent mindfulness group therapy plus aromatherapy massage at ten 120-min sessions while the control group received no intervention. Their pain perception, sleep quality, and quality of life as psychological functions were evaluated at baseline, at the end of intervention and 45 days later. Collected data were analyzed using the analysis of covariance method in SPSS v. 19 software.

Results The mindfulness therapy/aromatherapy massage significantly reduced the pain perception and its components and improved quality of life and sleep quality in the intervention group compared to the control group $(\mathrm{P}<0.05)$, and these effects remained stable in the follow-up phase.

Conclusion mindfulness therapy combined with aromatherapy massage improves the quality of life and sleep and reduces chronic pain in older women. It can be used as an effective intervention for improving general health and quality of life in older patients with chronic pain.

\section{Extended Abstract}

\section{Introduction}

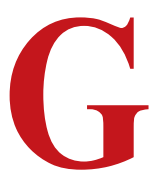

iven that the aging population in the world is increasing [1], paying attention to this age group psychologically and physically is important to have a healthy society. Since the elderly may have cognitive pain disorder is very common among this age group and can affect their quality of life [2], pain perception and sleep quality $[3,4]$, it is necessary to seek treatment for them to increase their mental and physical health, and it is better to use treatments that do not have the side effects caused by taking drugs. In this regard, mindfulness therapy along with massage can be one of the useful treatment methods. Therefore, the present study aimed to determine the simultaneous effect of mindfulness training and aromatherapy massage problems and unrealistic thoughts and given that chronic

\section{* Corresponding Author:}

Khadijeh Abolmaali Alhosseini, PhD.

Address: Department of Psychology, Faculty of Psychology, Roudehen Branch, Islamic Azad University, Roudehen, Iran.

Tel: +98 (21) 76505891

E-mail: abolmaali@riau.ac.ir 
on pain perception, sleep quality and quality of life in older women with chronic pain.

\section{Methods}

This is a quasi-experimental study with a pre-test, posttest, follow-up design using a control group. The study population consists of all women aged $\geq 60$ years with chronic pain referred to clinics, hospitals and rehabilitation centers in the west of Tehran, Iran in 2017 to receive physiotherapy services. The sample size was calculated 15 for each group based on Cohen's table considering effect size of 0.5 , test power of 0.9 and error rate of 0.05 [5]. After obtaining the necessary permits from the university and receiving the code of ethics, a convenience sampling was conducted using inclusion criteria (being female, having old age and chronic pain, and no cognitive impairment measured by MMSE test) and exclusion criteria (use of psychiatric drugs, receiving other therapies simultaneously, absence from two or more sessions of intervention, unwillingness to cooperate, not signing the consent form to participate in the study, and not completing the questionnaires). Finally, 30 women were selected and randomly assigned to two matched groups. The groups were evaluated for in terms of pain perception, sleep quality and quality of life using the Short Form-McGill Pain Questionnaire-2 (SF-MPQ-2), the Pittsburgh Sleep Quality Inventory (PSQI) and the WHO Quality of Life Questionnaire, Brief Version (WHOQOL$\mathrm{BREF}$ ), respectively.

The intervention group underwent 10 sessions of 120 minutes of mindfulness group therapy plus aromatherapy massage according to Kabat-Zinn's protocol [6] for 85 minutes and receiving aromatherapy massage training booklet prepared by Noavaran-e Salamat School and aromatic lavender essential oil for 35 minutes. The control group received no intervention. At the end of the intervention, the questionnaires were administered again to both groups. After 45 days, the questionnaires were completed again, and the collected data were analyzed by analysis of covariance (ANCOVA) in SPSS v. 19 software.

\section{Results}

Before performing the multivariate analysis of covariance, its assumptions were examined. The results of ShapiroWilks test showed that the distribution of data in the two groups was normal for the three study variables $(\mathrm{P}>0.05)$. The results of Levene's test were not significant $(\mathrm{P}>0.05)$ which confirmed the equality of variances. Hence, we were able to use parametric tests. The results of ANCOVA showed that the mean scores of post-test and follow-up were significantly different and the effect of group on sleep quality and quality of life was significant $\left(\mathrm{P}<0.05, \eta^{2}=0.154-0.343\right)$. In the follow-up stage, the results showed that the effect of group on the pain perception was significant $(\mathrm{P}<0.05)$ while the effect was not significant on the sleep quality and quality of life $(\mathrm{P}>0.05)$. Regarding the dimensions of pain perception, post-test results were significant in all dimensions except for intermittent pain, and the effect of group on all four dimensions of neuropathic pain, affective pain, continuous pain, and intermittent pain was significant $(\mathrm{P}<0.05)$. However, the effect of group in the follow-up stage on dimensions of continuous pain and intermittent pain was not significant $(\mathrm{P}<0.05)$. Finally, results showed that mindfulness therapy combined with aromatherapy massage significantly improved the perception of pain and its components, as well as the quality of life and sleep quality in older women in the intervention group compared to the control group, and these effects remained stable on the pain perception variable in the follow-up phase in patients.

\section{Discussion and Conclusion}

Simultaneous mindfulness therapy and aromatherapy massage reduce pain perception and improve sleep quality and quality of life in older women with chronic pain. Therefore, using massage techniques as a non-pharmacological strategy along with other treatment methods, along with teaching the patient to avoid negative thinking and pain-related rumination and have self-care skills through mindfulness reduce the severity of pain and cause relaxation in the elderly, limiting the possibility of complicated medical interventions and reducing the likelihood of functional disability. It is easy to use, safe, and cost-effective and is recommended in providing medical services to the elderly in the care centers.

\section{Ethical Considerations}

\section{Compliance with ethical guidelines}

All ethical principles were observed in this study. Informed consent was obtained from the participants after explaining the study method to them. They were assured of the confidentiality of their information and were free to leave the study at any time. This study obtained its ethical approval from the Islamic Azad University of Karaj Branch (Code: IR.IAU.K.REC.1397.51).

\section{Funding}

This study was extracted from the $\mathrm{PhD}$. dissertation of the first author at the Department of Health Psychology, Karaj Branch, Islamic Azad University, Karaj. 


\section{Authors' contributions}

All authors contributed equally in preparing this article

\section{Conflicts of interest}

The authors declare no conflict of interest.

\section{Acknowledgements}

The authors would like to thank all older adults participated in the study for their valuable cooperation as well as the Vice-Chancellor for Research of the Islamic Azad University of Karaj Branch 


\section{مقاله يثوهشى}

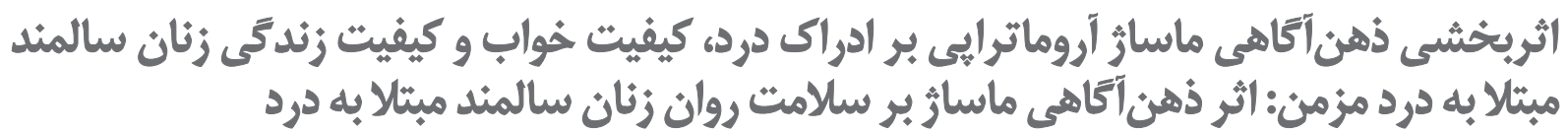

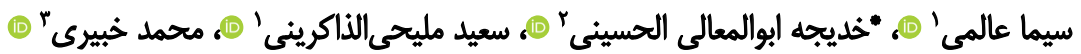

ا. تكروه رواثشناسى سلامت، واحد كرج، دائشكاه آزاد اسلامى، كرج، ايران.

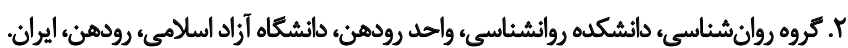

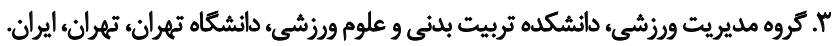

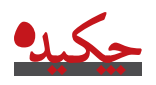

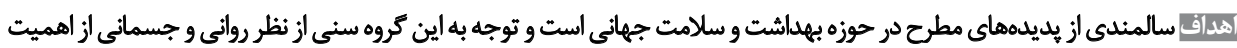

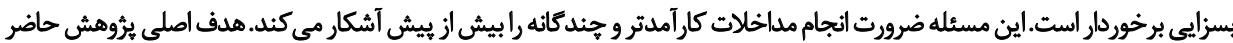

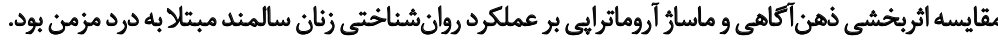

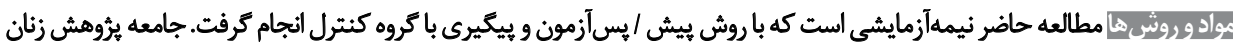

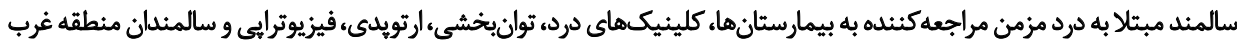

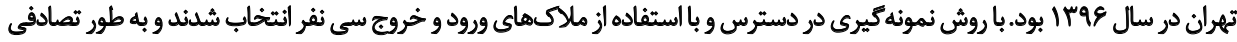

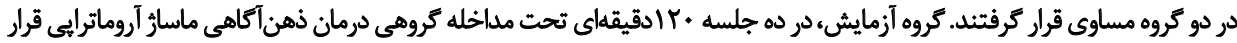

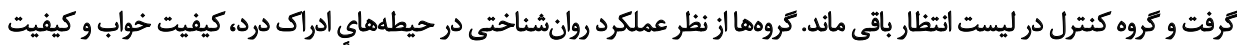

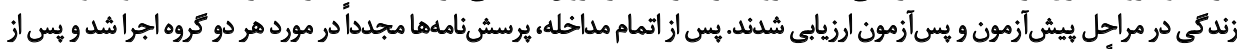

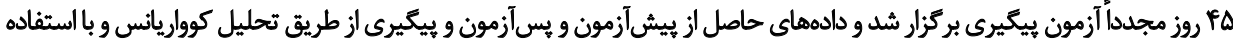

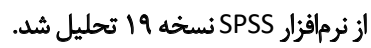

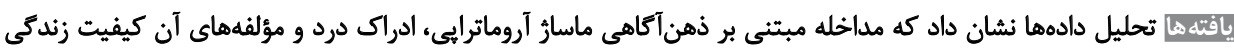

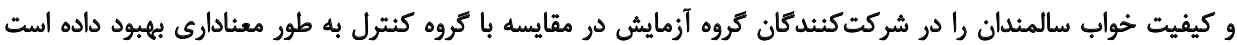
(P<+1•ه)

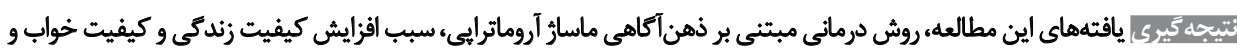

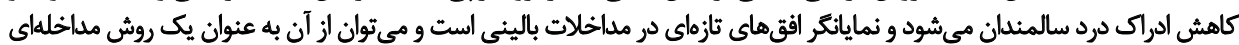

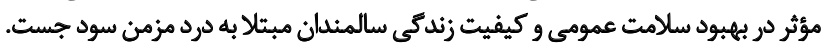

\author{
تاريخ دريافت: Tr شهريور

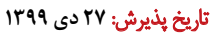

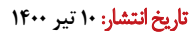

روانشناختى خاص همراه است. اين مشكلات هرجيند مانند

doleo

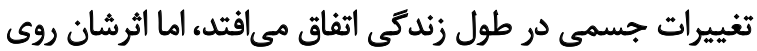

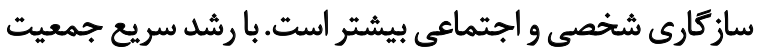

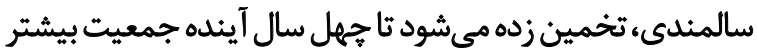

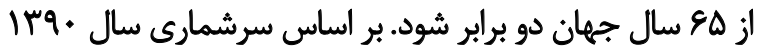

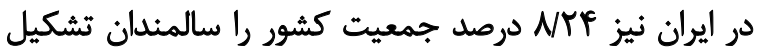

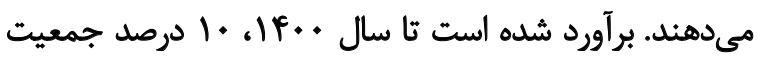
كشور را سالمندان تشكيل خواهند داد [1]].

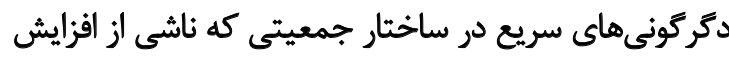

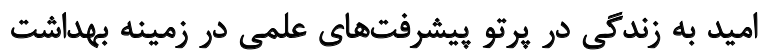

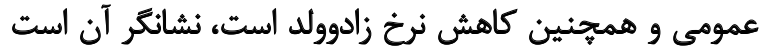

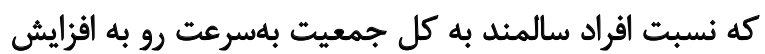

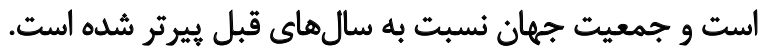

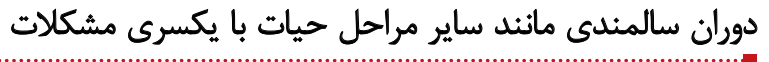

* نويسئده مسئول: دكتر خديجه ابوالمعالى الحسينى : نوريني

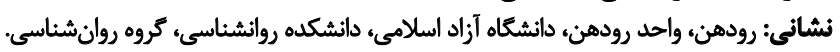
تلفن: نA abolmaali@riau.ac.ir يست الكترونيكي: 
بين بيشرفت درد و كيفيت خواب بيماران بزركسال مبتلا ارتباط

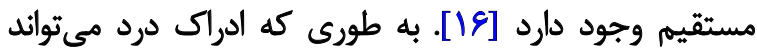

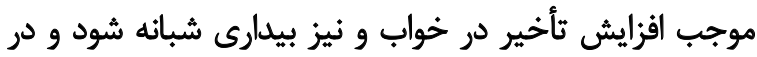

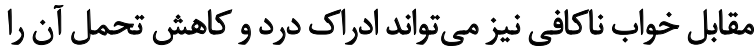

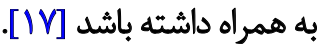

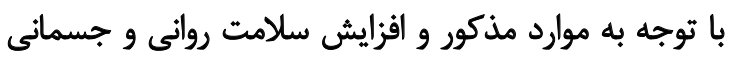

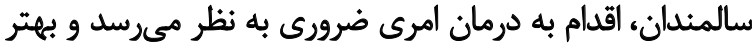

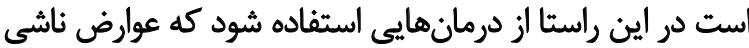

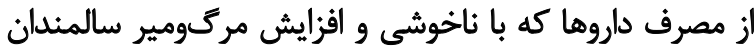

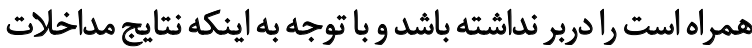

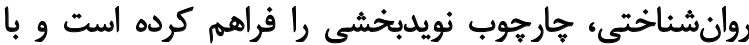

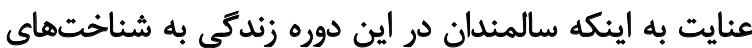

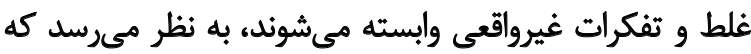

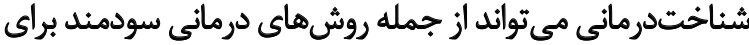

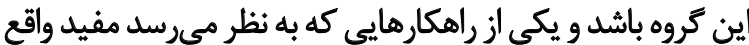

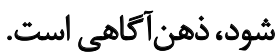

در رويكردهاى مبتنى بر ذهن آكاهى، درد به عنوان بخشى

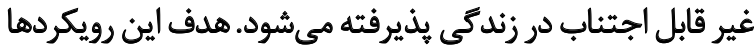

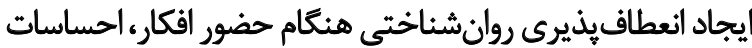

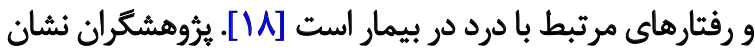

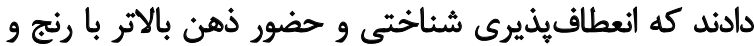

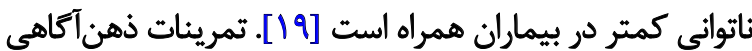

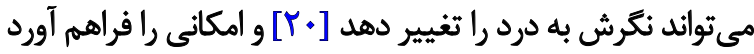

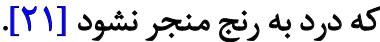

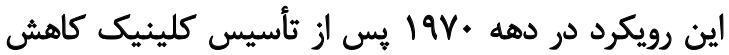

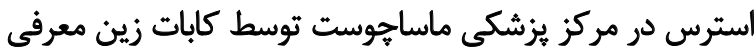

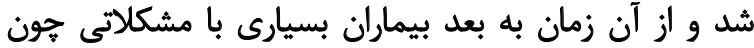
اختلالات خواب از اين روش بهره بردهاند [Tr] بـان.

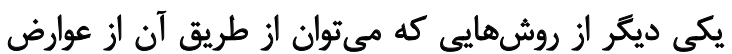

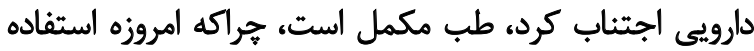

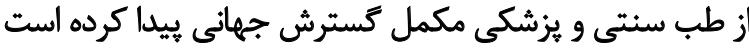

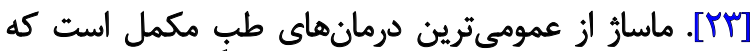

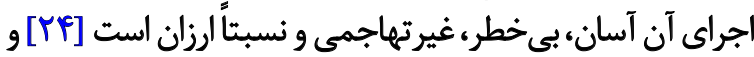

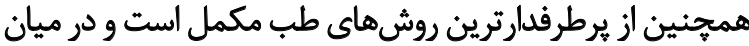

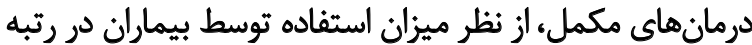

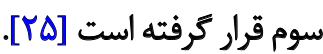

رايحهدرمانى' يكى از روشهاي ديكر طب مكمل است كه با بان

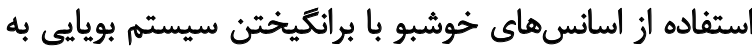

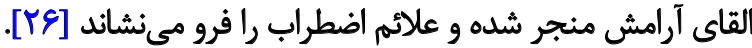

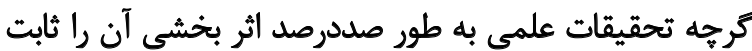
نكرداند، اما عقيده بر اين است كه رايحهدرمائى مى تواند اثرى اثنى

1. Aromatherapy

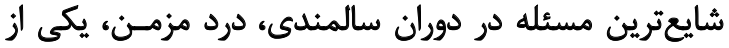

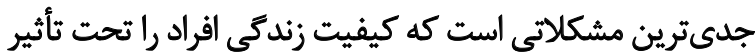

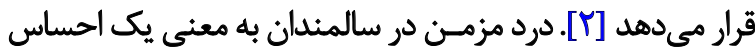

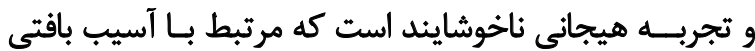

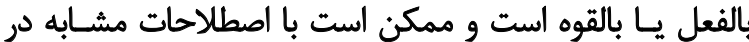

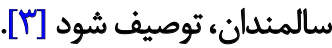

شايد درد عمومىترين فشار روانى باشد كه انسان با آن روبهرو

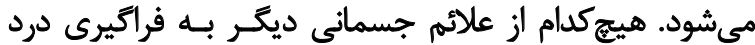

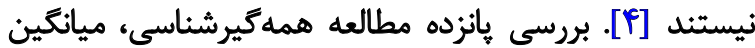

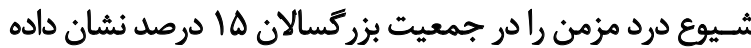

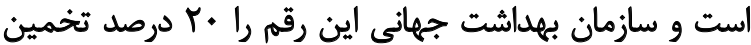

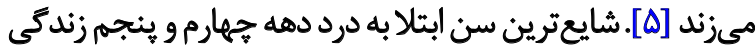

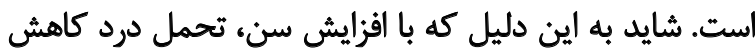

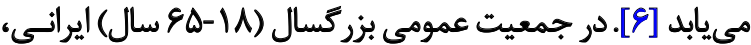

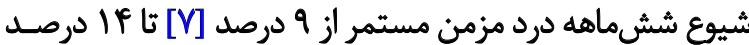

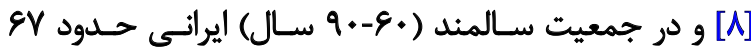

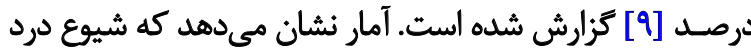

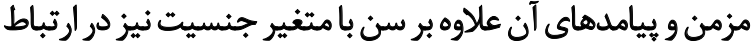

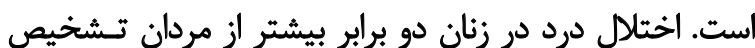

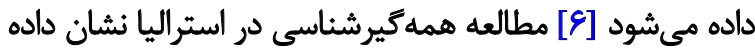

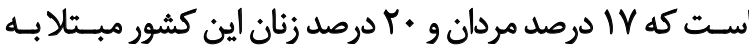

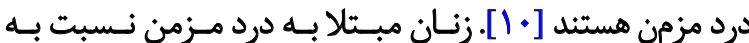

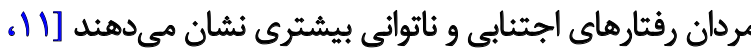

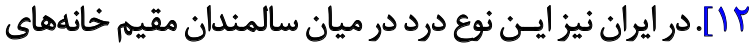

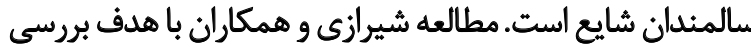

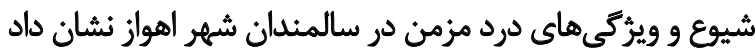

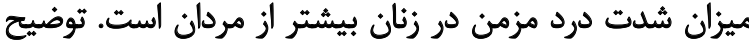

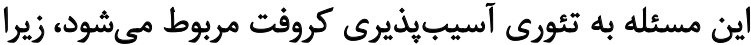
بر اساس اين ثئورى، زنان در مقايسه با مردان درئ مرئ مقابل درئ دردهاي

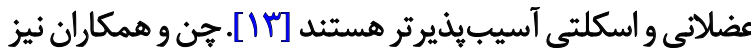

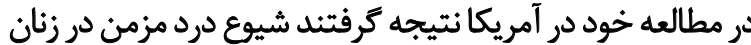

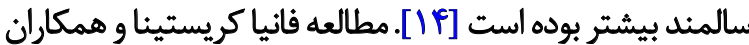

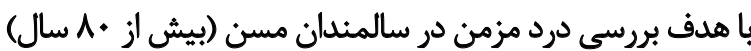

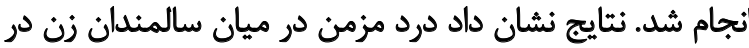

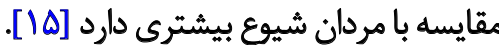

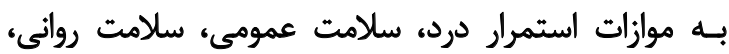

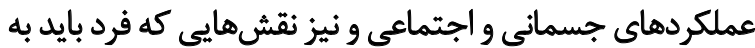

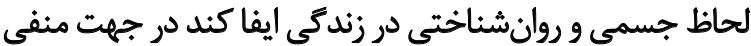

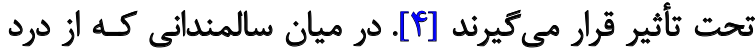

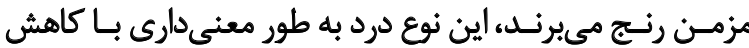

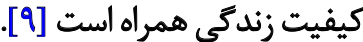

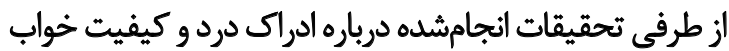

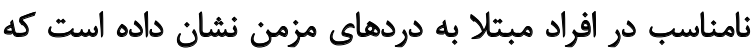


در دسترس انتخاب شدند كه به شيوه كمارش تصادفى (بر اساس

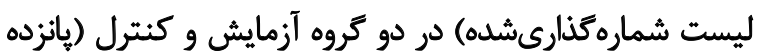

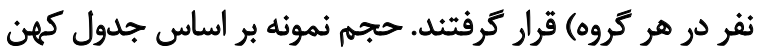

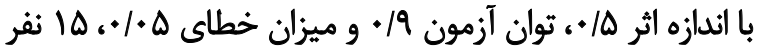

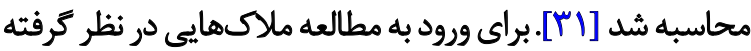

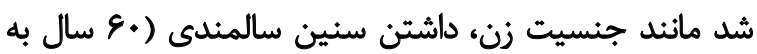

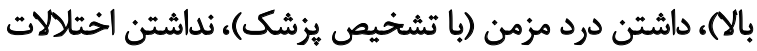

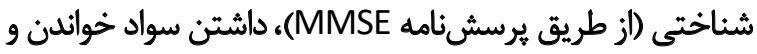

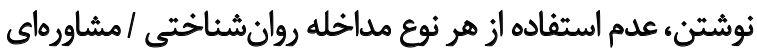

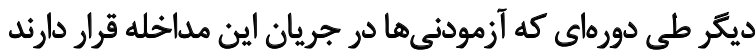

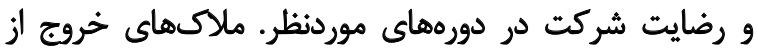

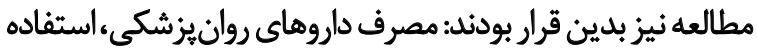

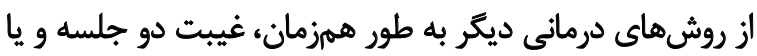

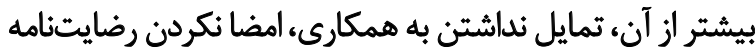

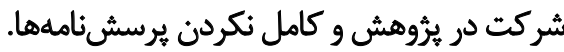

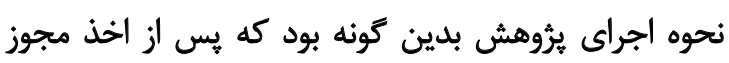

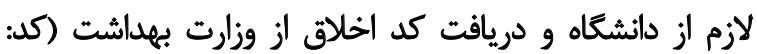

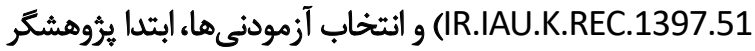

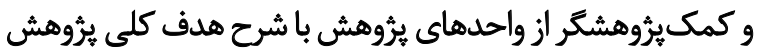

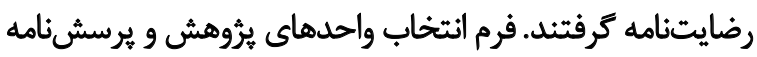

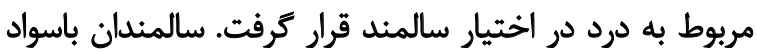

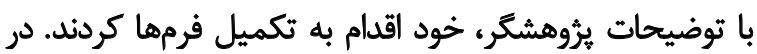

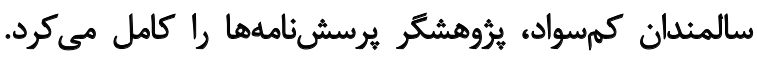

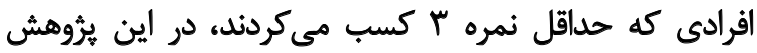

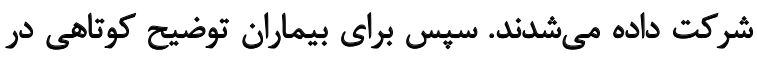

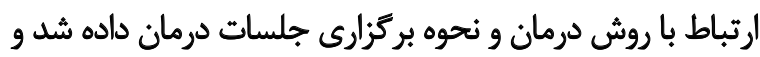

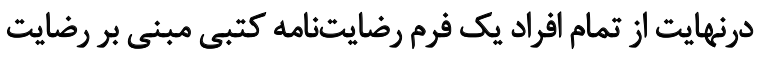

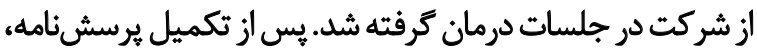

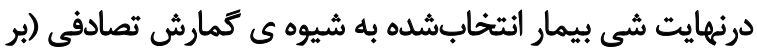

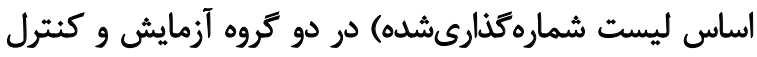

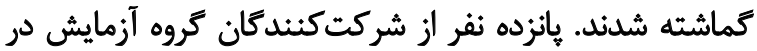

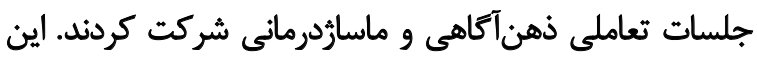

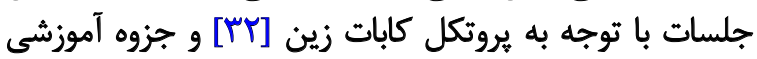

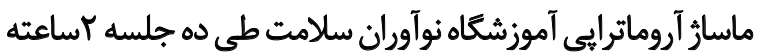

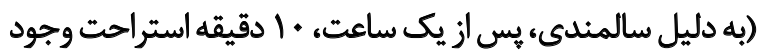

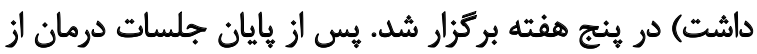

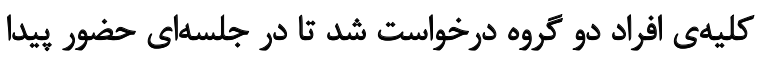

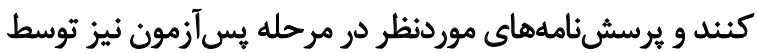

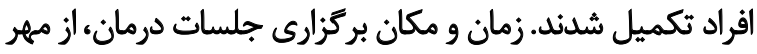

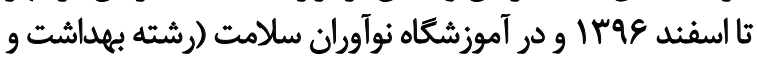

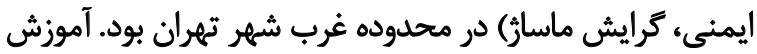

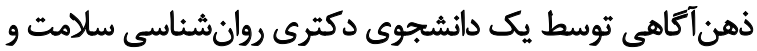

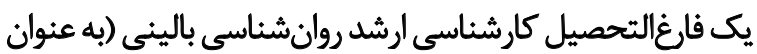

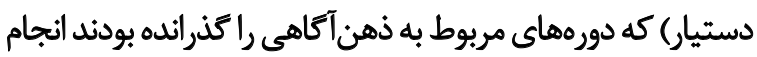

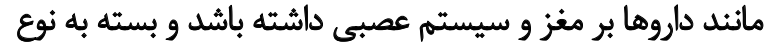

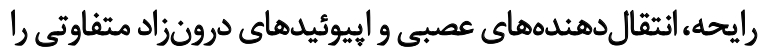

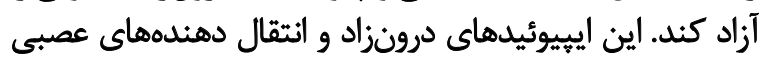

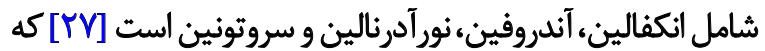

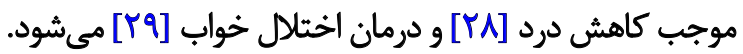

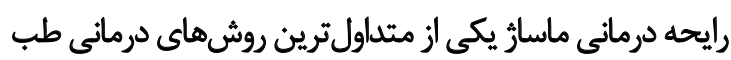

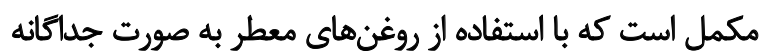

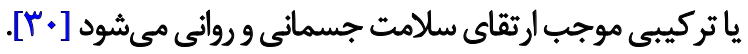

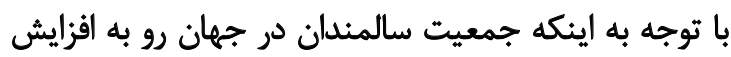

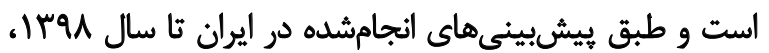

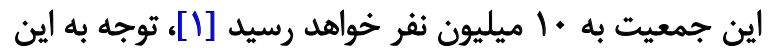

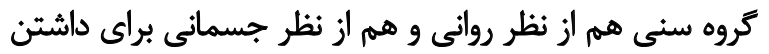

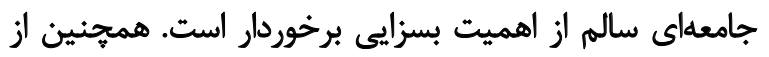

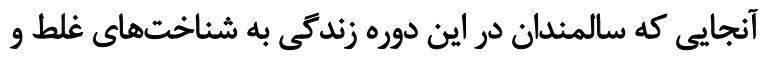

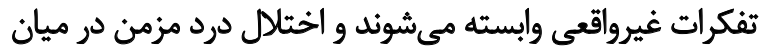

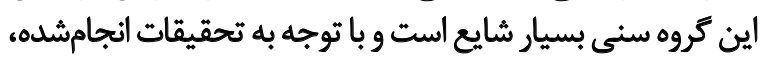

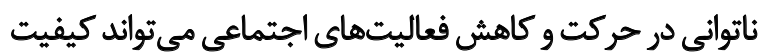

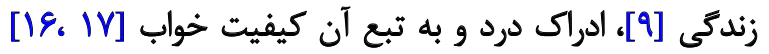

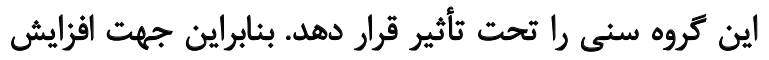

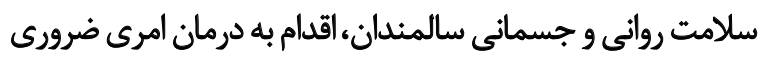

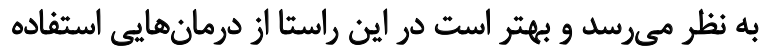

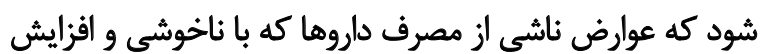

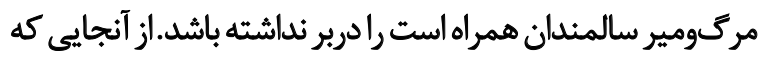

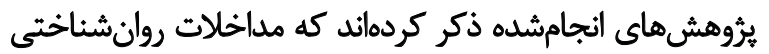

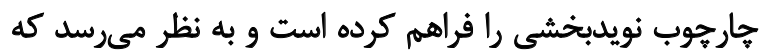

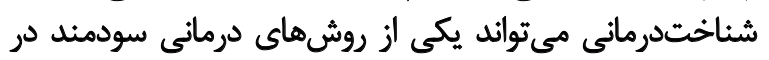

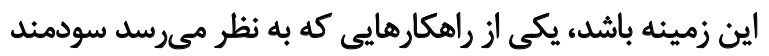

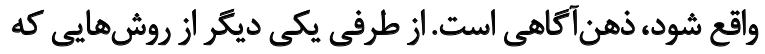

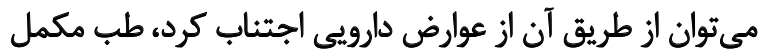

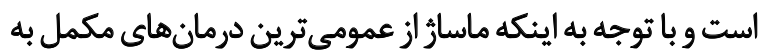

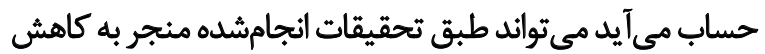

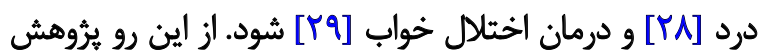

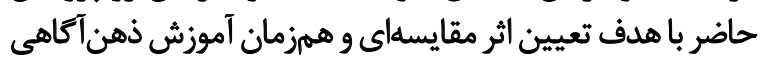

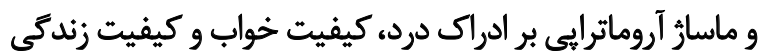

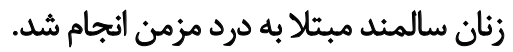

$$
\text { روث مطالعه }
$$

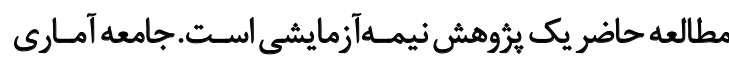

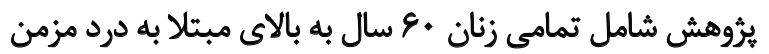

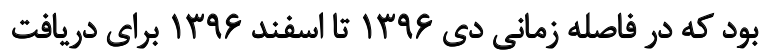

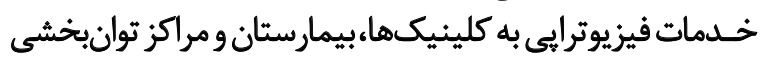

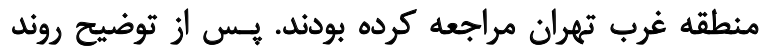

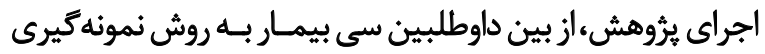




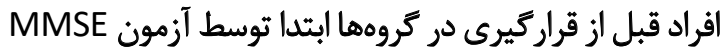

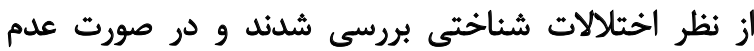

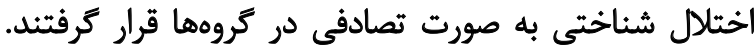

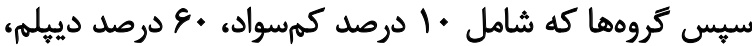

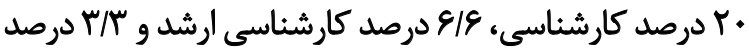

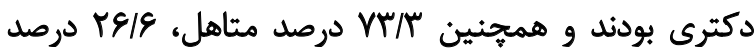

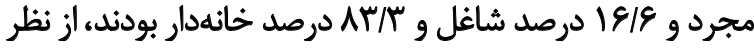

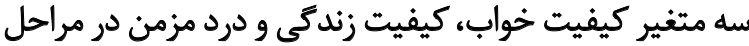

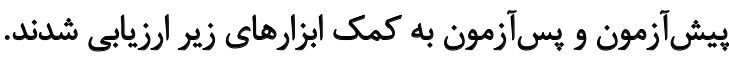

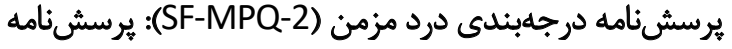

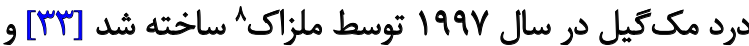

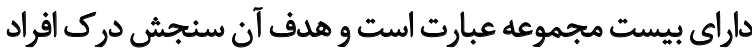

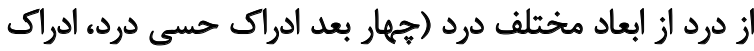

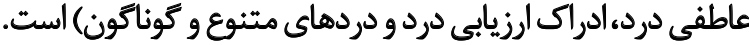

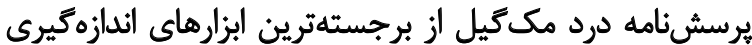

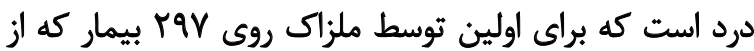

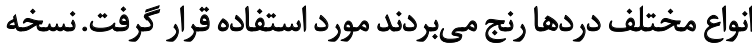
الصلاحشده يرسشنامه درد مكتكيل (SF-MPQ)نيز توسط

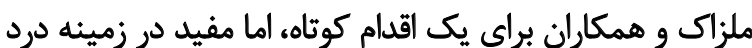

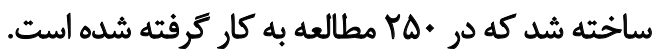

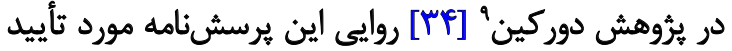

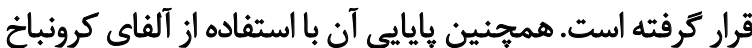

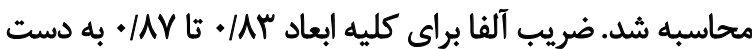

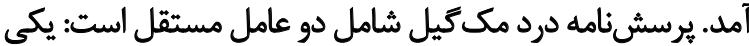

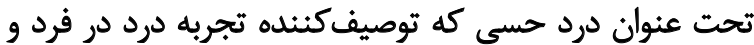

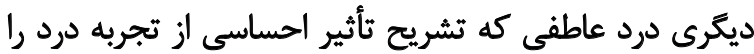

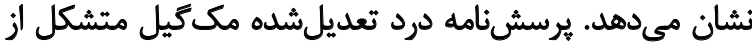

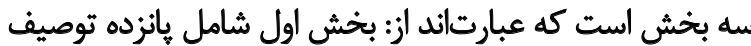

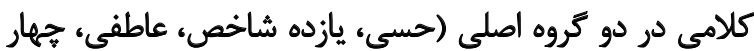

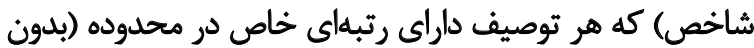

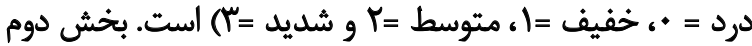

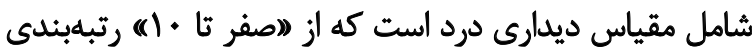

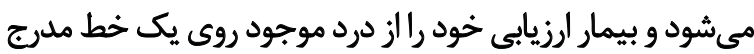

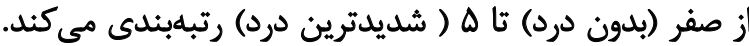

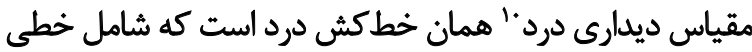

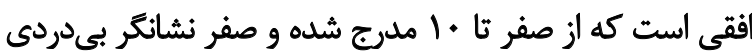

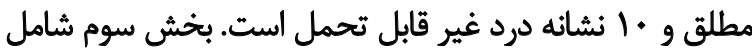

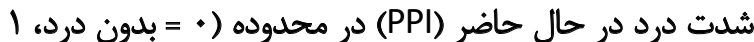

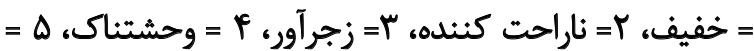
مشقتبار) است. مجموع نمرات كل درد بيمار برابر با نمره كل

\section{Melzack}

9. Dworkin

10. Visual Analogue Scale (VAS)

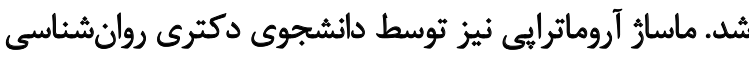

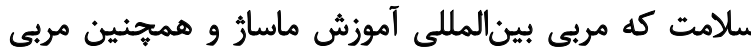
سازمان فنىوحرفهاى بود انجام يذيرفت.

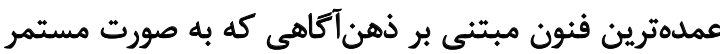

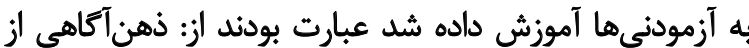

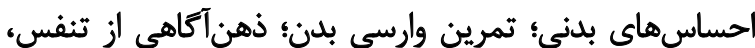

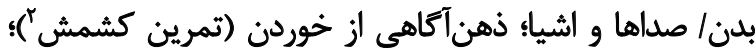

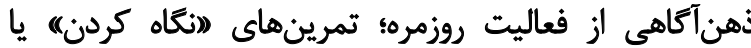

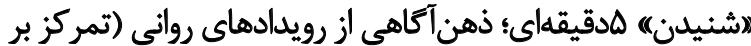

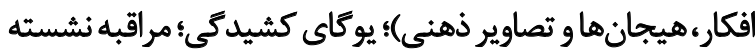

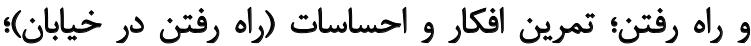

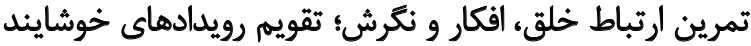

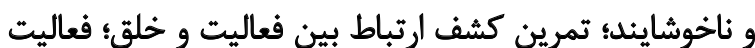

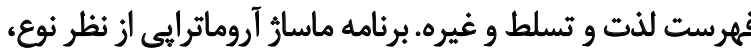

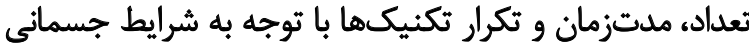

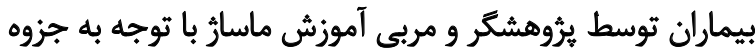

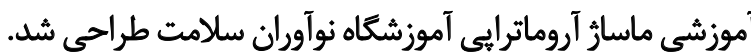

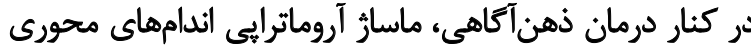

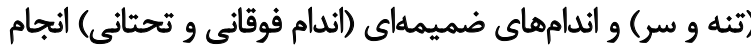

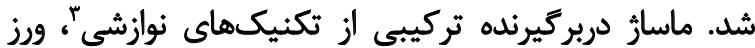

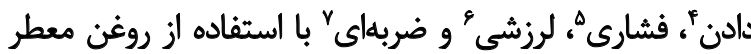

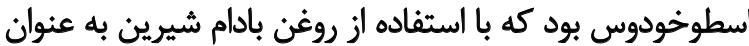

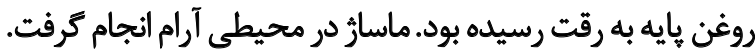

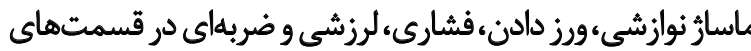

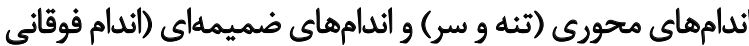

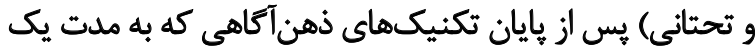

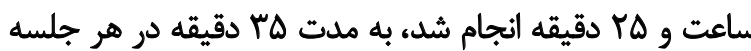

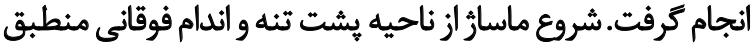

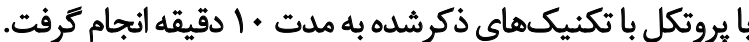

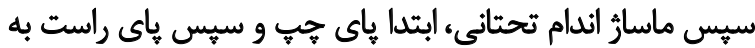

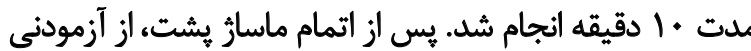

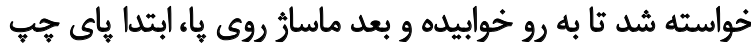

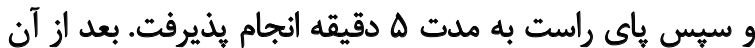

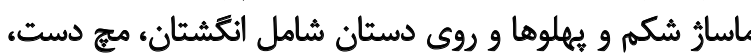

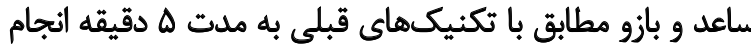

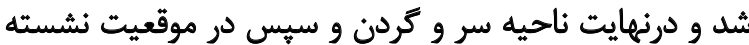

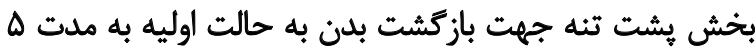
دقيقه انجام شد.

2. Raisin Practice

3. Stroking \& effleurage

4. Petrissage

5. Compression

6. Vibration

7. Tapotement 
قابل مشاهده است. بيش از انجام تحليل دادهها با روش تحليل

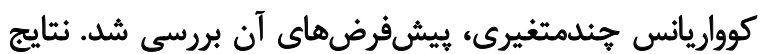

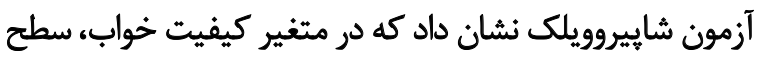

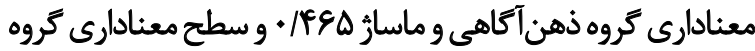

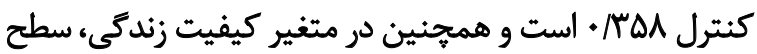

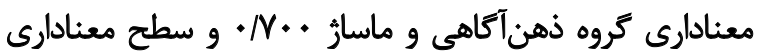

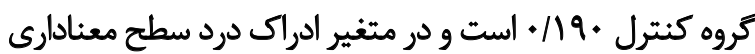

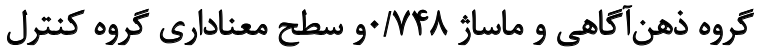

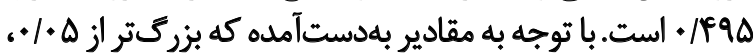

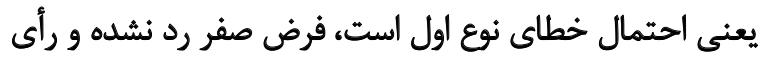

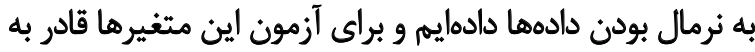
استفاده از آزمونهاي يارامتريك شديم داديم.

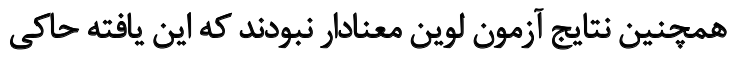

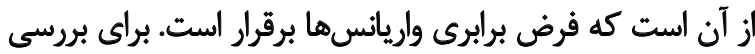

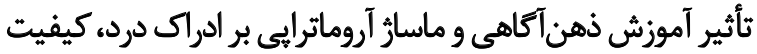

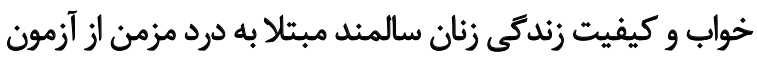

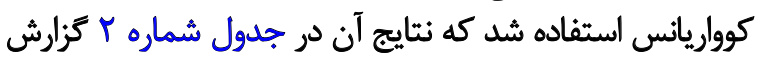

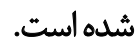

نتايج تحليل كوواريانس در جدول شماره ب نشان مي دهد

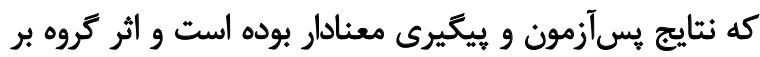

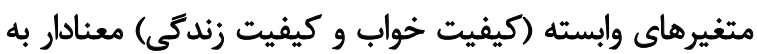

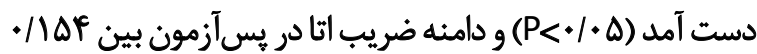

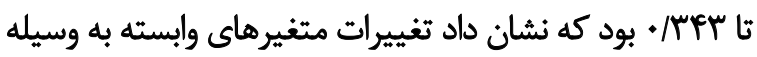

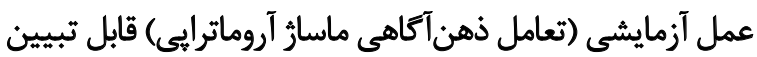

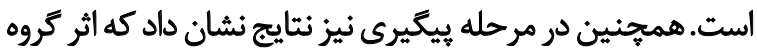

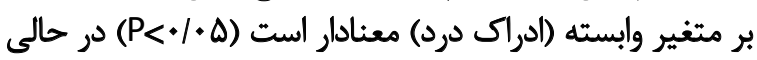

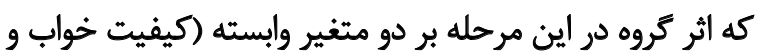

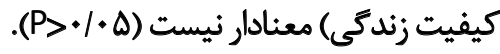

نتايج تحليل كوواريانس در جدول شماره بانشان مي دهد كهن نتايج

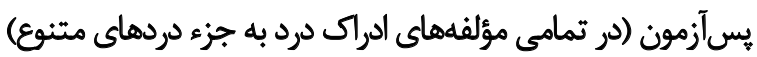

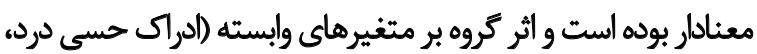

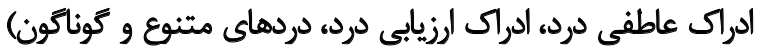

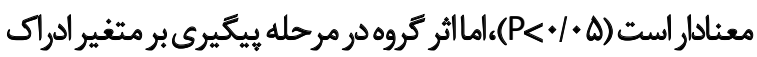

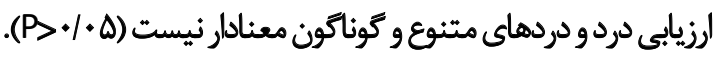

A

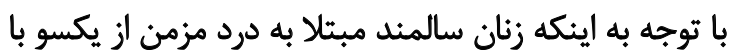

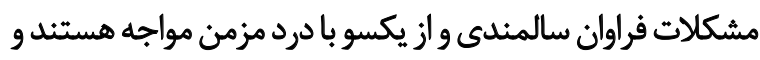

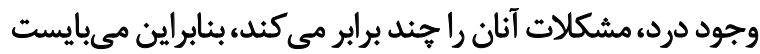

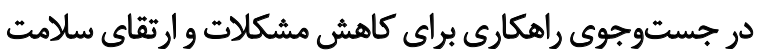

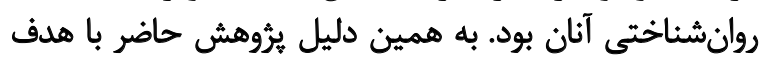

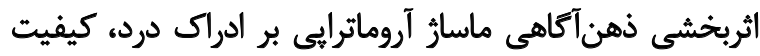

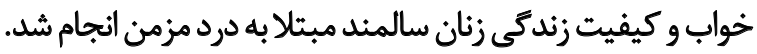

حاصلشده از كليه مجموعهها در ابعاد مختلف درد است.

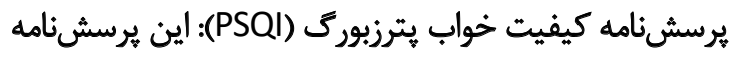

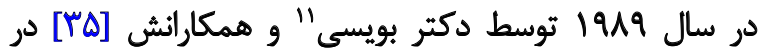

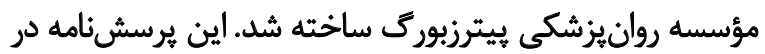

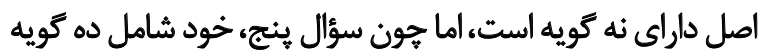

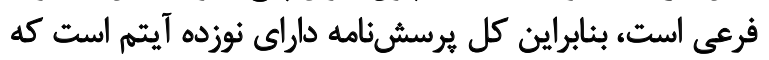

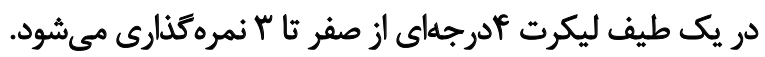

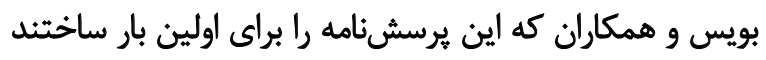

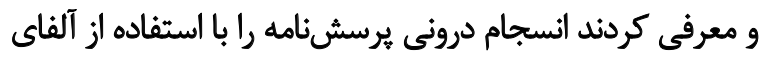

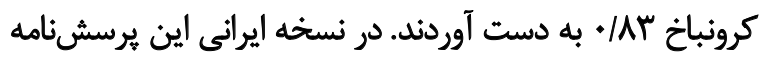

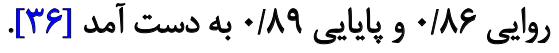

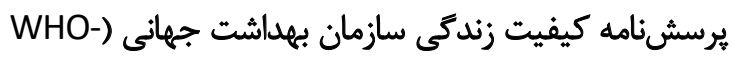
QQLL-BREF بعار WHOQOL-BREF

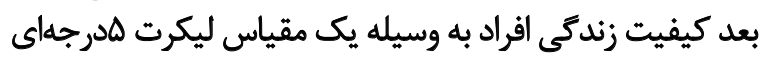

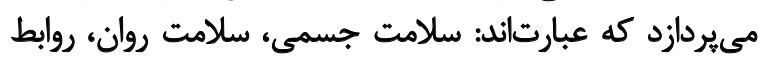

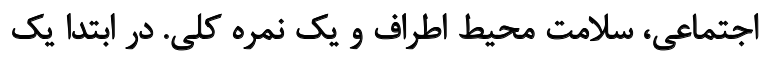

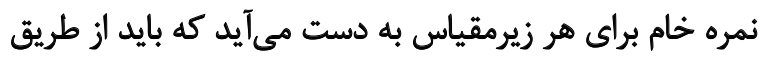

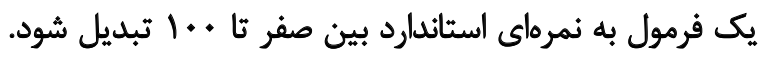

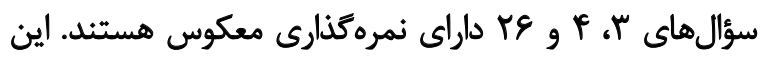

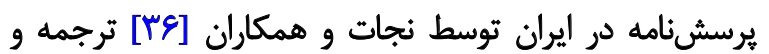

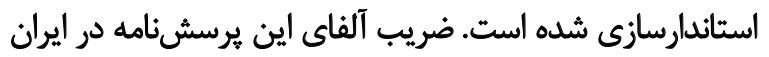

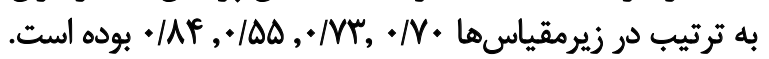

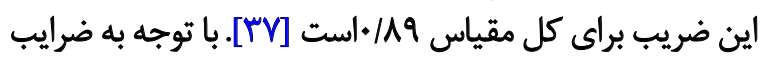

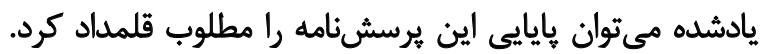

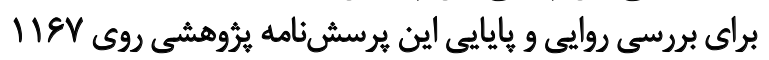

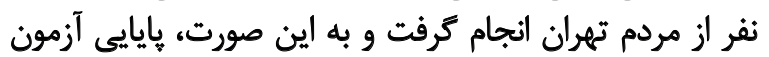

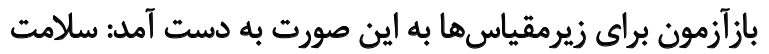

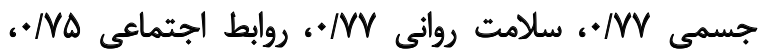

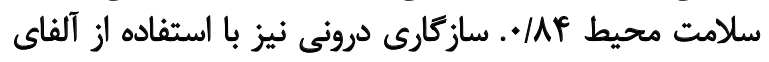

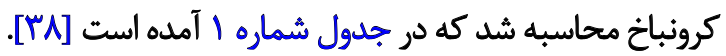

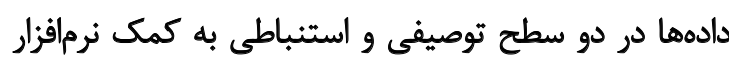

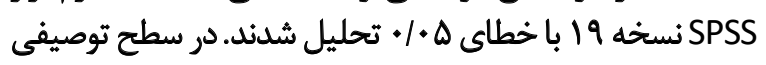

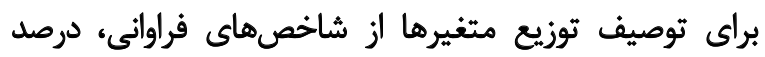

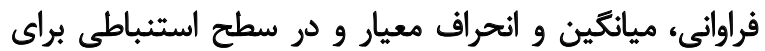
آزمون فرضهائ آمارى ازئن تحليل كوواريانس استفاده شد.

ياقثتهها

در جدول شماره ا، نتايج ميانكين و انحراف معيار متغيرهاى

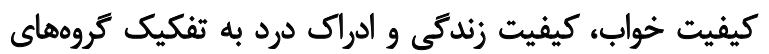

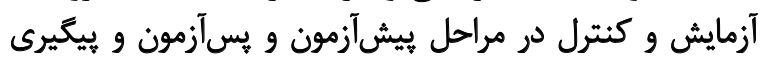


جدول ا. ميانكين و انحراف معيار متغيرهاى يُؤوهش در مراحل بيش آزمون، هس آزمون، ييكيرى

\begin{tabular}{|c|c|c|c|c|c|}
\hline بيشترين نمره & كمترين نمره & ميانكّين土|نحراف استاندارد & كروه & مرحله & متغير \\
\hline $\begin{array}{l}0 / \% \\
m q / .+\end{array}$ & $\begin{array}{l}\% \\
1 \mathrm{H} / .\end{array}$ & $\begin{array}{l}r \cdot / K A \pm R / M P \\
r V / T Y T \pm \Delta / \Delta q\end{array}$ & ذهن أكاهى و ماساز & ييش آزهون & \\
\hline $\begin{array}{l}\text { rel.. } \\
\text { rel.. }\end{array}$ & $\begin{array}{l}\text { W.. } \\
W \cdot \cdot\end{array}$ & $\begin{array}{l}10 / q V \pm V / T Y \\
Y Y / \circ \Phi \pm 1 \cdot / \pi r\end{array}$ & ذهن أكاهى و ماسارٌ & يس آلزمون & ادراى حسى درد \\
\hline $\begin{array}{l}m q \% . . \\
\mu q / . .\end{array}$ & $1 / \cdot$. & $\begin{array}{l}\mid r / F q \pm V / F q \\
r / / q \pm N \Delta f F\end{array}$ & ذهن أكاهي و ماسارٌ & ييكيرى & \\
\hline $\begin{array}{l}\mathrm{MP} / \mathrm{H} \\
\mathrm{rT/.}\end{array}$ & $\begin{array}{l}\mathrm{r} / \mathrm{H} / . . \\
\text { W. }\end{array}$ & $\begin{array}{l}V / N \Delta \pm r / \& \varepsilon \\
V / V Q \pm T / F E\end{array}$ & ذهن آكاهى و ماسارز & ييش آزمون & \\
\hline $\begin{array}{l}19 . . \\
19 . .\end{array}$ & $\begin{array}{l}\text { १/. } \\
\text { १/. }\end{array}$ & $\begin{array}{l}r / F \cdot \pm r / q q \\
N r q \pm r / m F\end{array}$ & ذهن آكاهى و ماساز & يس آزمون & ادراك عاطفى درد \\
\hline $\begin{array}{l}\mid Q / . . \\
|V| . .\end{array}$ & $\begin{array}{l}r \% \cdot . \\
ه / \cdot .\end{array}$ & $\begin{array}{l}r / A q \pm r / \Delta q \\
N \cdot \Delta \pm r / v \Delta\end{array}$ & ذهن آكاهى و ماسارّ & يبيكيرى & \\
\hline $\begin{array}{l}\mathrm{WV} \cdot . \\
\mathrm{Q} / \ldots\end{array}$ & $11 \%$ & $\begin{array}{l}r / r a \pm 1 / a . \\
r / R F \pm r / M\end{array}$ & ذهن أكاهي و ماسارً & ييش آزهون & \\
\hline$\Delta / \cdots$ & $\%$ & $\begin{array}{l}V / r \pm 1 / F q \\
r / P \& \pm 1 / N r\end{array}$ & ذهن آكاهى و ماسارز & يس آزمون & ادراى الرزيابي درد \\
\hline$\Delta / \cdot \bullet$ & $\begin{array}{l}.1 \\
.1\end{array}$ & 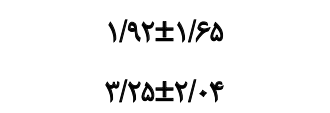 & ذهن آكاهى و ماسارٌ & ييكيرى & \\
\hline $\begin{array}{l}\Delta / \cdots \\
r+1+\infty\end{array}$ & $\begin{array}{l}.1 \\
4 / *+\end{array}$ & $\begin{array}{l}11 / r+ \pm \Delta / 81 \\
1+/ r \varepsilon \pm V / r A\end{array}$ & ذهن أكاهي و ماساز & ييشآزمون & \\
\hline $\begin{array}{l}19 \%+ \\
19 \%+\end{array}$ & $\begin{array}{l}\mathrm{V} / \cdot * \\
\mathrm{~V} / \cdot *\end{array}$ & $\begin{array}{l}\text { s/Qf } \pm r / \Delta . \\
1+/ 19 \pm V / r \Delta\end{array}$ & ذهن أكاهى و ماساز & يس آزمون & دردهاى كوناكون و متنوع \\
\hline IVI... & $\begin{array}{l}\psi / . . \\
q . .\end{array}$ & $\begin{array}{l}V / A \cdot \pm \Delta / \Delta \Delta \\
Q / Q \pm \& / Q Y\end{array}$ & ذهن آكاهى و ماسازٔ & ييكيرى & \\
\hline $\begin{array}{l}\text { W.. } \\
891 . .\end{array}$ & N... & $\begin{array}{l}P I / N A \pm \mid 1 / \cdot A \\
F \& / \Delta Y \pm|Y / F|\end{array}$ & ذهن آكاهى و هاسارٌ & ييش آزآهون & \\
\hline $\begin{array}{l}\text { efl.. } \\
\text { pq.. }\end{array}$ & $\begin{array}{l}r \Delta / \ldots \\
1 \% 1 . .\end{array}$ & $\begin{array}{l}r F /(\Lambda q \pm 9 / \Delta) \\
R T / \& V \pm 1 \cdot / \Delta \Delta\end{array}$ & ذهن أكاهي و هاساز & يس آزهون & ادراى درد (كل ثمره) \\
\hline $\begin{array}{l}801 . . \\
\Delta \% 1 .\end{array}$ & $\begin{array}{l}11 \%+. \\
F 1 / . .\end{array}$ & $\begin{array}{l}r e / v q \pm q / r \\
r / / 1 E \pm 1 \cdot / \Delta 1\end{array}$ & ذهن آكاهي و ماساز & بيكيرى & \\
\hline $\begin{array}{l}m \% \% \\
\mu \mathrm{m} / . .\end{array}$ & 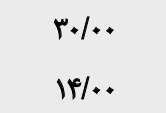 & $\begin{array}{l}r N G \pm \Psi / N \Delta \\
r \Psi / \varepsilon . \pm \Delta / 99\end{array}$ & ذهن أكاهي و ماسارز & ييش آزمون & \\
\hline $\begin{array}{l}\text { rIl.. } \\
\text { rN.. }\end{array}$ & Tr/.. & $\begin{array}{l}r V / T \Psi \pm T / R V \\
r r / q \cdot \pm T / \Delta .\end{array}$ & ذهن أكاهى و ماسازٔ & يس آزمون & كيفيت خواب \\
\hline $\begin{array}{l}r \% \% \\
r q 1 . .\end{array}$ & $\begin{array}{l}1 \% / . . \\
1.1 \%\end{array}$ & $\begin{array}{l}r \varphi / / r \pm F / r \varepsilon \\
r \Delta / \gamma^{\prime} \pm \& / g \varepsilon\end{array}$ & ذهن آكاهى و ماسازٔ & ييكيرى & \\
\hline
\end{tabular}




\begin{tabular}{|c|c|c|c|c|c|}
\hline بيشترين نمره & كمترين نمره & 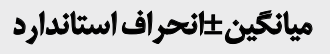 & كروه & مرحله & 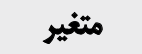 \\
\hline $\mathrm{me} / \mathrm{ot}^{\circ}$ & $|V|+*$ & $r e / q r \pm f / v q$ & ذهن أكاهى و ماسارّ & \multirow{2}{*}{ ييش آزمهون } & \multirow{6}{*}{ كيفيت زندكى } \\
\hline M.. & $8 \pi / .$. & $V \Delta / V^{\mu} \pm V / T^{\mu}$ & 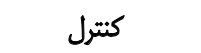 & & \\
\hline$\Delta F / \infty$ & $v \cdot 1 .$. & $r q / T V \pm r / s \Delta$ & ذهن أكاهى و ماساز & \multirow{2}{*}{ يس آزّمون } & \\
\hline$A F \%$ & gr/.. & $V E / \cdot . \pm E / M T$ & 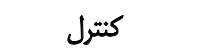 & & \\
\hline$q \% /$. & D1/.. & $V E / \Delta r \pm I V / \Delta r$ & ذهن|"كاهي و ماسارٔ & \multirow{2}{*}{ يكيرى } & \\
\hline $91 / .$. & gr/.. & $V T / T \cdot \pm \& / g r$ & كنترل - & & \\
\hline
\end{tabular}

20

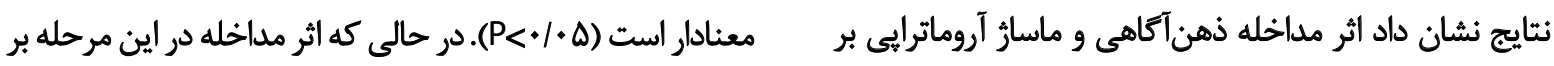

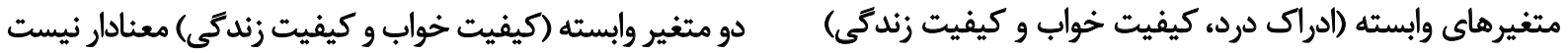

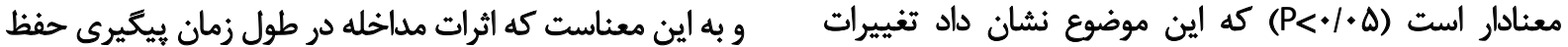

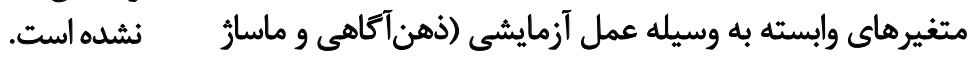

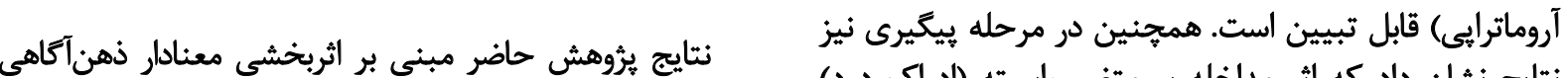

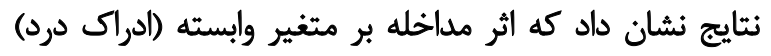
جدول r. نتايج تحليل كوواريانس تأثير تعاملى ماسارً آروماترايى و ذهن آكاهى بر ميزان نمرات كيفيت خواب، كيفيت زندكى و ادراك درد در كروه آزمايش و كنترل

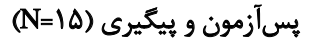

\begin{tabular}{|c|c|c|c|c|c|c|c|}
\hline مجذور اتا & معنادارى سطح & Fاره F F F & مجذوراتين & أزاديه & مجذورات & منيع & متغير \\
\hline & .10 & $11 / N e 9$ & ITE. IAAT & $r$ & $F \cdot A T / \Delta \Delta \Delta$ & يس آز هون & \multirow{2}{*}{ ادراك درد نمره كل } \\
\hline.$/ M T$ &...$r$ & $\Delta / 194$ & explier & r & $1997 / 0 \cdot r$ & ييكيرى & \\
\hline.$/ 191$ & $.1 \cdot 11$ & elrev & $P+|\Delta P|$ & 1 & $P=|\Delta P|$ & يسآزهون & \multirow{2}{*}{ كيفيت خواب } \\
\hline 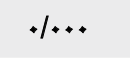 & . /aur & $.1 . .1$ &.$/ . Y$. & 1 & $.1 . Y$. & ييكيرى & \\
\hline$r V+1$. & $+\infty \%$ & $210 / 9$ & . rI/MYP & 1 & . TI/TME & يس آزمون & \multirow{2}{*}{ كيفيت زندكى } \\
\hline $.1 . \Delta$ & - /rar & $\mid(M M \mid$ & $\Lambda \Delta / N \cdot \varepsilon$ & 1 & $V+8 / 1 \Delta$ & ييكيرى & \\
\hline
\end{tabular}

ile

جدول ب. نتايج تحليل كوواريانس تأثير تعاملى ماساز آروماترايى و ذهن آكاهى بر نمرات مؤلفهاى ادراك درد در كروه آزمايش و كنترل يس آزمون و بيكيرى $(\mathrm{N} \equiv \backslash \Delta)$

\begin{tabular}{|c|c|c|c|c|c|c|c|}
\hline مجذور اتا & معادارى & أماره F & مجزياتكين & آزاديك & مجزدورات & منيع & متغير \\
\hline . NPA & $+|r|$ & $r / \wedge 19$ & rra/rir & $r$ & EAVIFTA & يسأزمون & \multirow{2}{*}{ ادراك حسى درد } \\
\hline.$/ N T$ & $+1 \cdot 0$ & $\mathrm{r} / \Delta \mathrm{W}$ & $189 / 719$ & $r$ & $\Delta T V / Q \Delta S$ & ييكيرى & \\
\hline . Mert & 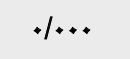 & $1 . / \mathrm{Rr}$ & NENEq & r & $r \varepsilon \cdot / r+\varphi$ & يسأزمون & \multirow{2}{*}{ ادراى عاطفى درد } \\
\hline.$/ M F$ & $+1 *+1$ & $8 / .1 Y$ & Al/frF & $r$ & $M F F / M r I$ & ييكيرى & \\
\hline.$/ 118$ & $\% A r$ & T/TMQY & Q/DHT & $r$ & TNAY & يسأزمون & \multirow{2}{*}{ ادراك ارزيابى درد } \\
\hline .1 .49 & - Rro & I/TYA & $r / \ldots$ & 1 & $f / \ldots$ & ييكيرى & \\
\hline.$/ 1 \Delta q$ & \% & $r / F W$ & IST/FTq & $r$ & FAV/YNE & يسأزمون & \multirow{2}{*}{ دردهاى متنوع و كوناكون } \\
\hline . &.$/ E I r$ & .181 & $r \Delta / F A I$ & $r$ & ve/pitr & يبكيرى & \\
\hline
\end{tabular}


با توجه به محتواى جلسات ذهن آكاهى مبتنى بر كاهش

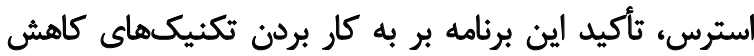

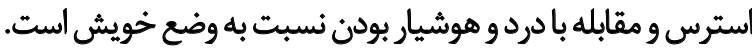

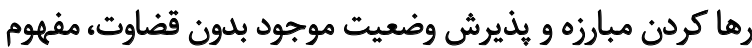

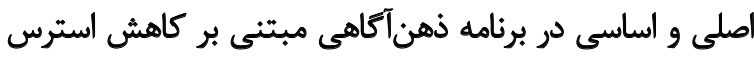

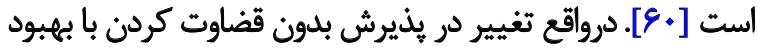

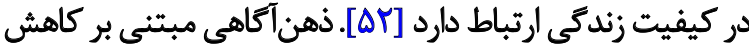

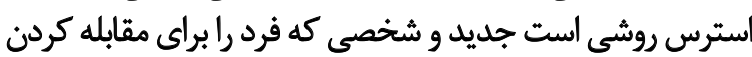

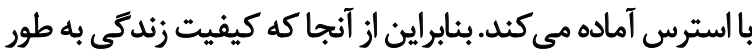

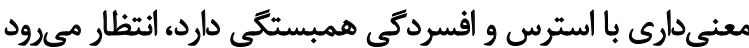

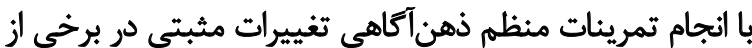

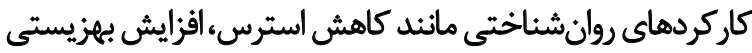

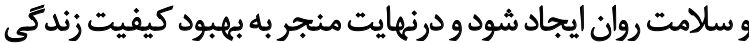

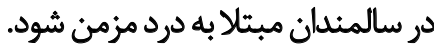

همجنين دليل عدم همسويى اين يُروهش با يُروهش عالمى

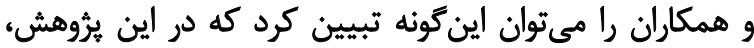

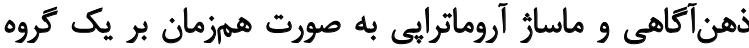

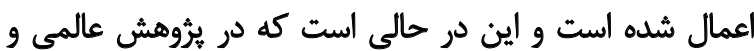

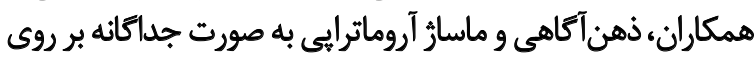

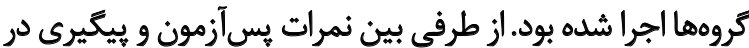

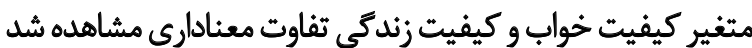

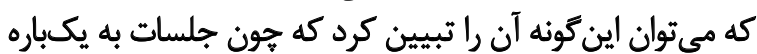

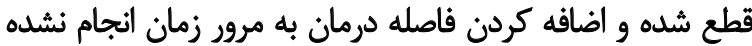

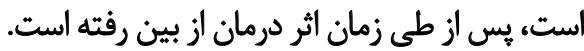

باتوجه به نتايج بهدست آمده، درمان همزمان ذهن آكاهي مانى ماسار

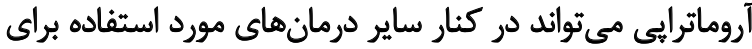

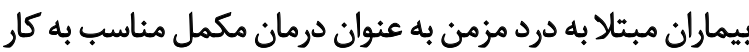

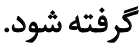

\section{نتيجليَّيرىنهايى}

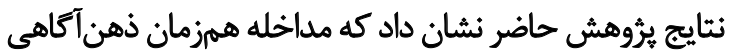

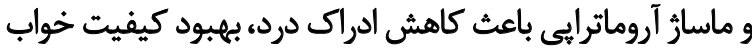

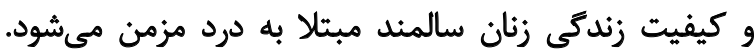

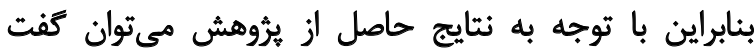

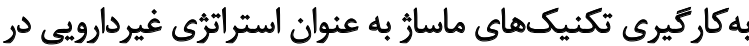

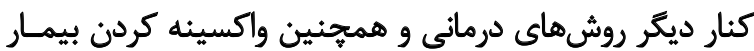

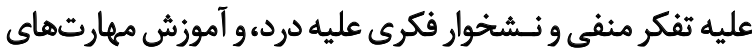

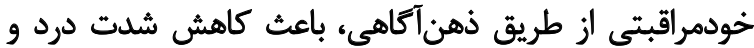

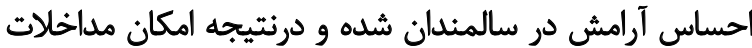

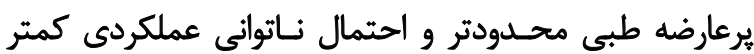

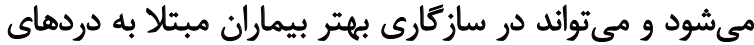

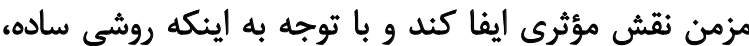

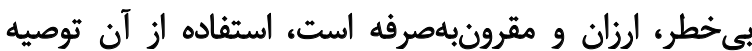

و ماسار آروماترايى بر ادراك درد با نتايج يثروهشهاي ديكر كه

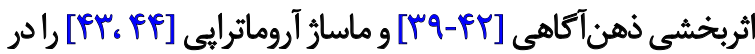

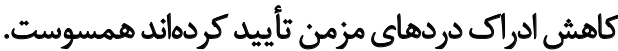

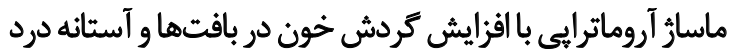

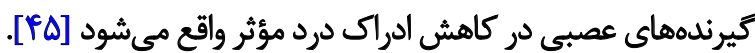

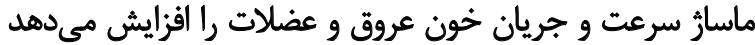

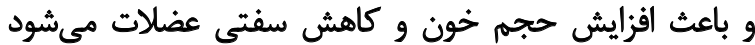

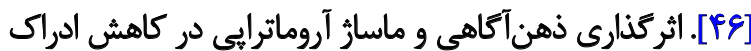

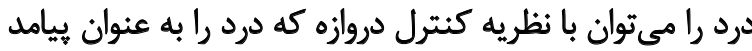

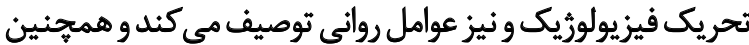

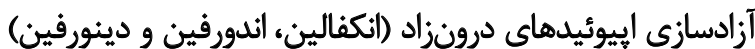

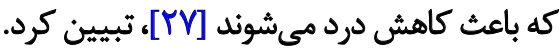

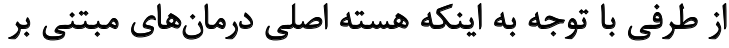

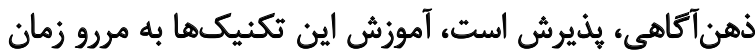

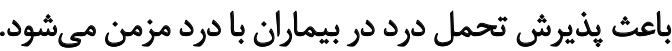

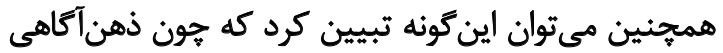

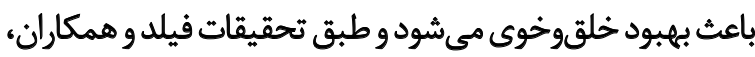

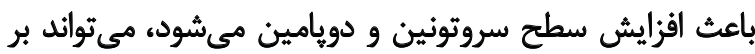

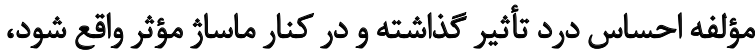

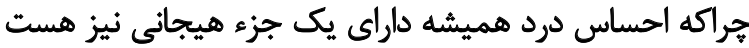

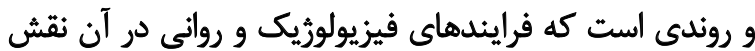

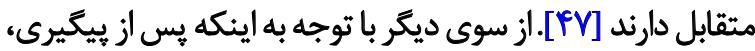

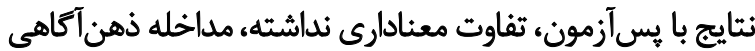

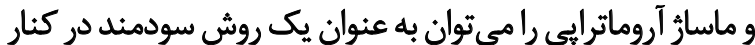

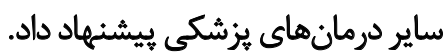

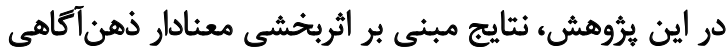

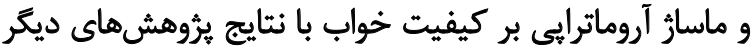

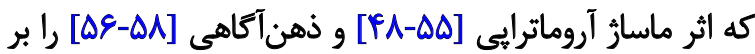

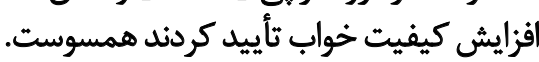

اين يافتهها را مي توان اين كونه تبيين كرد كه با توجه به اينكه

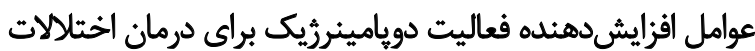

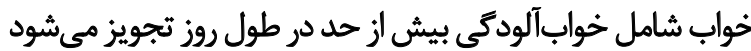

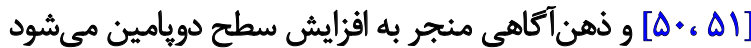

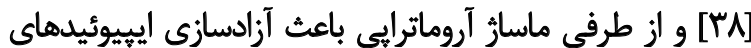

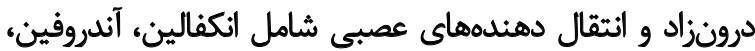

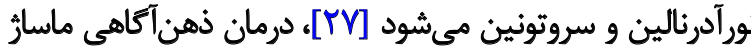
آروماترايى مى تواند بر كيفيت خواب مؤثر واقع شود.

همجنين نتايج يُووهش حاضر مبنى بر اثربخشى معناديار

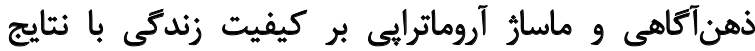

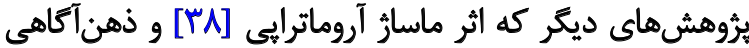

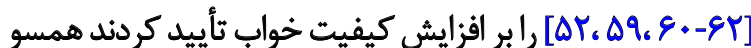

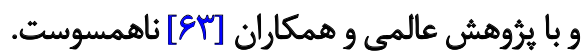


مىشود. نتايج اين تحقيق مىتواند به منظور حفظ و ارتقاى

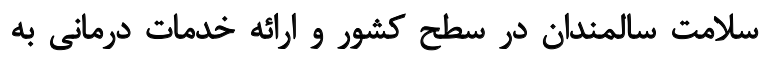

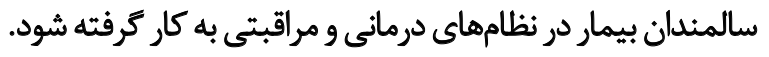

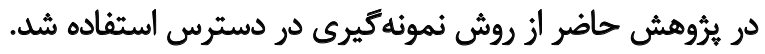

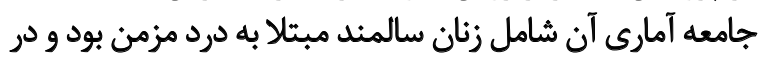

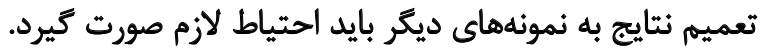

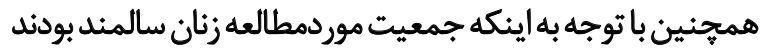

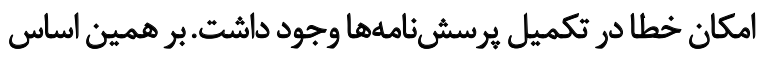

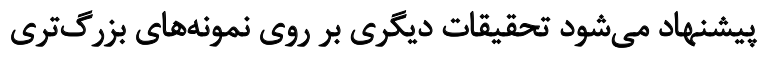

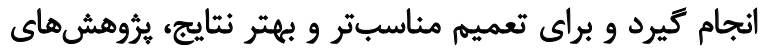

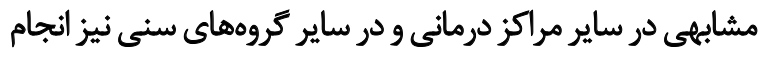

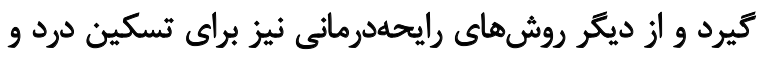

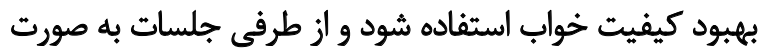

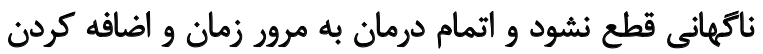
فاصله درمان صورت يذيرد.

مالاحظات اخلاقي

\section{ييروى از اصول انفاق يؤوهش}

اصول اخلافى تماماً در اين مقاله رعايت شده است. شركت

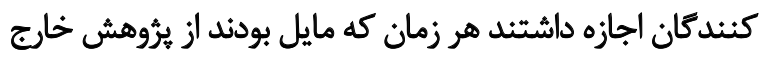

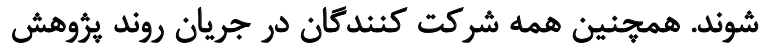

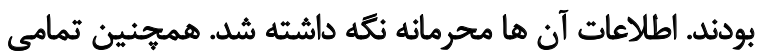

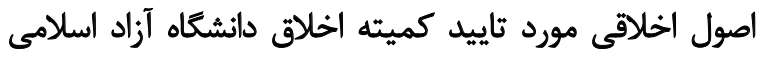

واحدكرج قرار كرفته است (كد: IR.IAU.K.REC.1397.51).

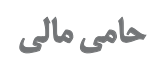

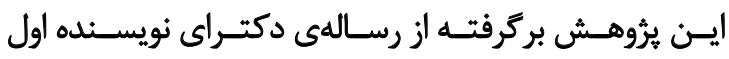

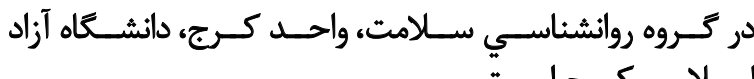
اسـلامي، كـرج استـت

$$
\text { مشار كت نويسندكان }
$$

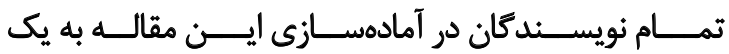

اندازه مشـــــاركت داشــــــاند.

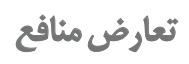

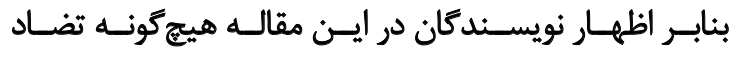

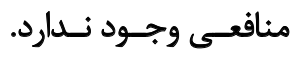

$$
\text { تشكر و قدردانى }
$$

از معاون محترم يُروهشى دانشعاه آزاد اسلامى كرج و استادان

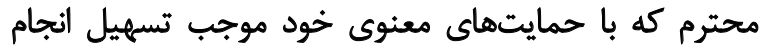
مطالعه شدند، قدردانى مي شمايتهود. 


\section{References}

[1] Fotoukian Z, Mohammadi Shahboulaghi F, Fallahi Khoshknab M. [Analytical on empowerment interventions in older people with chronic disease: A review literature (Persian)]. Journal of Health Promotion Management. 2013; 2(4):65-76. http://jhpm.ir/ article-1-239-en.html

[2] Iliaz R, Ozturk GB, Akpinar TS, Tufan A, Sarihan I, Erten N, et al. Approach to pain in the elderly. Journal of Gerontology \& Geriatric Research. 2013; 2(3):1000125. https://www.longdom.org/openaccess/approach-to-pain-in-the-elderly-2167-7182.1000125.pdf

[3] Kaye AD, Baluch A, Scott JT. Pain management in the elderly population: A review. The Ochsner Journal. 2010; 10(3):179-87. [PMID] [PMCID]

[4] Gatchel Robert J. Psychological approaches to pain management: a practitioner's handbook. [Asghari Moghaddam MA, Najarian B, Mohammadi M, Dehghani M, Persian trans.]. Tehran: Roshd; 2002. http://opac.nlai.ir/opac-prod/bibliographic/ 645630

[5] Turk DC, Swanson K. Efficacy and cost-effectiveness treatment for chronic pain: An analysis and evidence-based synthesis. In: Schatman ME, Campbell A, Loeser JD, editors. Chronic Pain Management: Guidelines for Multidisciplinary Program Development. Boca Raton: CRC Press; 2007. pp. 15-38. [DOI:10.3109/9781420045130-2]

[6] Mohammadi F, Mohammadkhani P, Dolatshahi B, Asghari MA [The effects of "mindfulness meditation for pain management" on the severity of perceived pain and disability in patients with chronic pain (Persian)]. Salmand: Iranian Journal of Ageing. 2011; 6(1):59-66. http://salmandj.uswr.ac.ir/article-1-378-en.html

[7] Asghari Moghaddam MA, Karami B, Rezaei S. [The prevalence rates of lifetime pain and chronic pain in two small cities in Iran (Persian)]. Journal of Psychology. 2002; 6(1):30-51. https:/ / www. sid.ir/fa/JOURNAL/ViewPaper.aspx?id=21987

[8] Asghari Moghaddam MA. [The prevalence rate of chronic pain and some of its associations among the employees of a big industrial company in Tehran (Persian)]. Daneshvar Raftar. 2004; 11(4):1-14. http://cpap.shahed.ac.ir/article_2544.htm

[9] Asghari A, Ghaderi N, Ashory A. The prevalence of pain among residents of nursing homes and the impact of pain on their mood and quality of life. Archives of Iranian Medicine. 2006; 9(4):368-73. [PMID]

[10] Blyth FM, March LM, Brnabic AJM, Jorm LR, Williamso M, Cousins MJ. Chronic pain in Australia: A prevalence study. Pain. 2001; 89(2):127-34. [DOI:10.1016/S0304-3959(00)00355-9]

[11] Philips HC, Jahanshahi M. The components of pain behavior report. Behaviour Research and Therapy. 1986; 24(2):117-25. [DOI:10.1016/0005-7967(86)90082-3]

[12] Keefe FJ, Lefebvre JC, Egert JR, Affleck G, Sullivan MJ, Caldwell DS. The relationship of gender to pain, pain behavior, and disability in osteoarthritis patients: The role of catastrophizing. Pain. 2000; 87(3):325-34. [DOI:10.1016/S0304-3959(00)00296-7]

[13] Shirazi M, Manoochehri H, Zagheri Tafreshi M, Zayeri F, Alipour V. [Prevalence of chronic pain and its characteristics among elderly people in Ahvaz city: A cross sectional study (Persian)] Journal of Geriatric Nursing. 2015; 2(1):62-78. http:/ / ign.medilam. ac.ir/article-1-159-en.html

[14] Chen Q, Hayman LL, Shmerling RH, Bean JF, Leveille SG. Characteristics of chronic pain associated with sleep difficulty in older adults: The Maintenance of Balance, Independent Living, Intellect, and Zest in the Elderly (MOBILIZE) Boston study.
Journal of the American Geriatrics Society. 2011; 59(8):1385-92. [DOI:10.1111/j.1532-5415.2011.03544.x]

[15] Santos FC, de Moraes NS, Pastore A, Cendoroglo MS. [Chronic pain in long-lived elderly: Prevalence, characteristics, measurements and correlation with serum vitamin D level (EnglishPortuguese)]. Revista Dor. 2015; 16(3):171-5. [DOI:10.5935/18060013.20150034]

[16] Kaye AD, Baluch AR, Kaye RJ, Niaz RS, Kaye AJ, Liu H, et al. Geriatric pain management, pharmacological and nonpharmacological considerations. Psychology \& Neuroscience. 2014; 7(1):1526. [DOI:10.3922/j.psns.2014.1.04]

[17] World Health Organization. The world health report 2008: Primary health care: Now more than ever [Internet]. 2008 [Updated 2008 October 1]. Available from: https://reliefweb.int/report/ world/world-health-report-2008-primary-health-care-nowmore-ever

[18] Eggermont LHP, Leveille SG, Shi L, Kiely DK, Shmerling RH Jones RN, et al. Pain characteristics associated with the onset of disability in older adults: The maintenance of balance, independent living, intellect, and zest in the Elderly Boston Study. Journal of the American Geriatrics Society. 2014; 62(6):1007-16. [DOI:10.1111/jgs.12848]

[19] Silver JK. Chronic pain and the family: A new guide. Cambridge: Harvard University Press; 2009. https://books.google com/books?id=mcOaqNRcq3YC\&dq

[20] Roy R. Chronic pain and family: A clinical perspective. New York: Springer Science \& Business Media; 2006. https:/ /books. google.com/books?id=XtFXzPBSCXIC\&dq

[21] Riahi ME. [A comparative study on the status of elderly in the traditional and modern societies (Persian)]. Salmand: Iranian Journal of Ageing. 2008;3(3-4):10-21. http:/ / salmandj.uswr.ac.ir/ article-1-96-en.html

[22] Finan PH, Goodin BR, Smith MT. The association of sleep and pain: An update and a path forward. The Journal of Pain. 2013 14(12):1539-52. [DOI:10.1016/j.jpain.2013.08.007]

[23] Berk M. Sleep and depression - theory and practice. Australian Family Physician. 2009; 38(5):302-4. [PMID]

[24] Crombez G, Vlaeyen JWS, Heuts PHTG, Lysens R. Pain-related fear is more disabling than pain itself: Evidence on the role of pain-related fear in chronic back pain disability. Pain. 1999; 80(1):329-39. [DOI:10.1016/S0304-3959(98)00229-2]

[25] Dahl J, Lundgren T. Acceptance and Commitment Therapy (ACT) in the treatment of chronic pain. In: Baer RA, editor. Mindfulness-Based Treatment Approaches Clinician's Guide to Evidence Base and Applications, Practical Resources for the Mental Health Professional. Burlington, MA: Academic Press; 2006. pp. 285-306. [DOI:10.1016/B978-012088519-0/50014-9]

[26] Narimani M, Ariapooran S, Abolghasemi A, Ahadi B. [Effectiveness of mindfulness-based stress reduction and emotion regulation training in the affect and mood of chemical weapons victims (Persian)]. Journal of Arak University Medical Sciences. 2012 15(2):107-18. http://jams.arakmu.ac.ir/article-1-922-en.html

[27] Salimzade O, Pourhosein R, Lavasani M. Investigating the effectteaching mindfulness-based stress reduction in reducing pschological symptoms in adolescent boys in dorms. International Journal of Behavioral Social and Movement Sciences. 2014; 3(4):44-58. http://ijobsms.in/6dvol03issue004p7.html

[28] Goldin PR, Gross JJ. Effects of Mindfulness-Based Stress Reduction (MBSR) on emotion regulation in social anxiety disorder. Emotion. 2010; 10(1):83-91. [DOI:10.1037/a0018441] 
[29] Best TM, Hunter R, Wilcox A, Haq F. Effectiveness of sports massage for recovery of skeletal muscle from strenuous exercise. Clinical Journal of Sport Medicine. 2008; 18(5):446-60. [DOI:10.1097/JSM.0b013e31818837a1]

[30] Sherman KJ, Cherkin DC, Kahn J, Erro J, Hrbek A, Deyo RA, Eisenberg DM. A survey of training and practice patterns of massage therapists in two US states. BMC Complementary and Alternative Medicine. 2005; 5:13. [DOI:10.1186/1472-68825-13]

[31] Sarmad Z, Bazargan A, Hejazi E. [Research methods in behavioral science (Persian)]. $1^{\text {st }}$ ed. Tehran: Aghah; 1997. http:// opac.nlai.ir/opac-prod/bibliographic/555496

[32] Kabat-Zinn Jon. Full catastrophe living: Using the wisdom of your body and mind to face stress, pain, and illness. New York, United States: Delacorte Press; 1990. https://books. google.com/books?id=QeGGPwAACAAJ\&dqhttps:/ / books.google.com/books?id=i4AedPJKtYYC\&dq

[33] Dworkin RH, Turk DC, Revicki DA, Harding G, Coyne KS, Peirce-Sandner $S$, et al. Development and initial validation of an expanded and revised version of the Short-form McGill Pain Questionnaire (SF-MPQ-2). Pain. 2009; 144(1):35-42. [DOI:10.1016/j.pain.2009.02.007]

[34] Heidari AR, Ehteshamzadeh P, Marashi M. [The relationship between insomnia intensity, sleep quality, sleepiness and mental health disorder with educational performance in female adolescences (Persian)]. Scientific Research Quarterly of Woman and Culture. 2010; 2(4):65-76. http:/ /jwc.iauahvaz. ac.ir/article_523533.html

[35] Buysse DJ, Reynolds III CF, Monk TH, Berman SR, Kupfer DJ. The Pittsburgh sleep quality index: A new instrument for psychiatric practice and research. Psychiatry Research. 1989; 28(2):193-213. [DOI:10.1016/0165-1781(89)90047-4]

[36] Nejat S, Montazeri A, Holakouie Naieni K, Mohammad K, Majdzadeh S. [The World Health Organization Quality of Life (WHOQOL-BREF) questionnaire: Translation and validation study of the Iranian version (Persian)]. Journal of School of Public Health and Institute of Public Health Research. 2006; 4(4):1-12. http:/ / sjsph.tums.ac.ir/article-1-187-en.html

[37] Nejati V, Ashayeri H. [Health related quality of life in the elderly in Kashan (Persian)]. Iranian Journal of Psychiatry and Clinical Psychology. 2008; 14(1):56-61. http:/ /ijpcp.iums. ac.ir/article-1-415-en.html

[38] Plews-Ogan M, Owens JE, Goodman M, Wolfe P, Schorling J. Brief report: A pilot study evaluating mindfulness-based stress reduction and massage for the management of chronic pain. Journal of General Internal Medicine. 2005; 20(12):1136-8. [DOI:10.1111/j.1525-1497.2005.0247.x]

[39] Swain N, Lennox Thompson B, Gallagher S, Paddison J, Mercer S. Gratitude Enhanced Mindfulness (GEM): A pilot study of an Internet-delivered programme for self-management of pain and disability in people with arthritis. The Journal of Positive Psychology. 2020; 15(3):420-6. [DOI:10.1080/17 439760.2019.1627397

[40] Allone C, Corallo F, Scarlata F, Formica C, Cartella E, Lo Buono $\mathrm{V}$, et al. Mindfulness interventions and pain management in a patient with encephalomyelitis. Complementary Therapies in Clinical Practice. 2019; 36:69-71. [DOI:10.1016/j. ctcp.2019.06.001]

[41] Firoozi M, Rouhi Sh. Couple therapy based on mindfulness to improve the satisfaction of life and pain self-efficacy in pa- tients with chronic pain. The Open Pain Journal. 2020; 13:1-6 [DOI:10.2174/1876386302013010001]

[42] Marchand WR. Mindfulness-based stress reduction, mindfulness-based cognitive therapy, and Zen meditation for depression, anxiety, pain, and psychological distress. Journal of Psychiatric Practice. 2012; 18(4):233-52. [DOI:10.1097/01. pra.0000416014.53215.86]

[43] Cheatham SW, Stull KR. Roller massage: Comparison of three different surface type pattern foam rollers on passive knee range of motion and pain perception. Journal of Bodywork and Movement Therapies. 2019; 23(3):555-60. [DOI:10.1016/j.jbmt.2019.05.002]

[44] Nunes GS, Bender PU, de Menezes FS, Yamashitafuji I, Vargas VZ, Wageck B. Massage therapy decreases pain and perceived fatigue after long-distance Ironman triathlon: A randomised trial. Journal of Physiotherapy. 2016; 62(2):83-7. [DOI:10.1016/j.jphys.2016.02.009]

[45] Ayik C, Özden D. The effects of preoperative aromatherapy massage on anxiety and sleep quality of colorecta surgery patients: A randomized controlled study. Complementary Therapies in Medicine. 2018; 36:93-9. [DOI:10.1016/j. ctim.2017.12.002]

[46] Dubrovsky V. The effect of massage on athletes' cardiorespiratory systems. Soviet Sports Review. 1990; 25:36-8. https:// scholar.google.com/scholar?hl=en\&as_sdt $=0 \% 2 \mathrm{C} 5 \& \mathrm{q}=\mathrm{The}+$ effect + of + massage + on + athletes $\% 27+$ cardiorespiratory + syste ms\&btnG=

[47] Sarafino EP, Smith TW. Health psychology: Biopsychosocial interactions. Hoboken: John Wiley \& Sons; 2014. https:/ / books.google.com/books?id=ypODBgAAQBAJ\&dq

[48] Seo SY, Chang SY. [Effects of aroma hand massage on sleep depression and quality of life in the institutionalized elderly women (Korean)]. Korean Journal of Women Health Nursing 2009; 15(4):372-80. [DOI:10.4069/kjwhn.2009.15.4.372]

[49] Yang HJ, Kang HY, Kim IS. The effects of aroma foot reflex massage on sleep, depression and problem behaviors on elderly with dementia. Korean Journal of Adult Nursing. 2011; 23(6):574-83. https://www.koreamed.org/SearchBasic.php? $\mathrm{RID}=1094 \mathrm{KJAN} / 2011.23 .6 .574 \& \mathrm{DT}=1$

[50] Qu WM, Xu XH, Yan MM, Wang YQ, Urade Y, Huang ZL. Essential role of dopamine $\mathrm{D}_{2}$ receptor in the maintenance of wakefulness, but not in homeostatic regulationof sleep, in mice. Journal of Neuroscience. 2010; 30(12):4382-9. [DOI:10.1523/JNEUROSCI.4936-09.2010]

[51] Lu J, Jhou TC, Saper CB. Identification of wake-active dopaminergic neurons in the ventral periaqueductal gray matter. Journal of Neuroscience. 2006; 26(1):193-202. [DOI:10.1523/ JNEUROSCI.2244-05.2006]

[52] Nyklíček I, Kuijpers KF. Effects of mindfulness-based stress reduction intervention on psychological well-being and quality of life: Is increased mindfulness indeed the mechanism? Annals of Behavioral Medicine. 2008; 35(3):331-40. [DOI:10.1007/ s12160-008-9030-2

[53] Kashani F, Kashani P. The effect of massage therapy on the quality of sleep in breast cancer patients. Iranian Journal of Nursing and Midwifery Research. 2014; 19(2):113-8. [PMID] [PMCID]

[54] Shinde MB, Anjum Sh. Effectiveness of slow back massage on quality of sleep among ICU patents. International Journal of 
Science and Research. 2014; 3(3):292-8. https://www.ijsr.net/ get_abstract.php?paper_id=20131124

[55] Oshvandi Kh, Abdi S, Karampourian A, Moghimbaghi A, Homayonfar Sh. The effect of foot massage on quality of sleep in ischemic heart disease patients hospitalized in CCU. Iranian Journal of Critical Care Nursing. 2014; 7(2):66-73. http://jccnursing. com/article-1-279-en.pdf

[56] Carlson LE, Garland SN. Impact of Mindfulness-Based Stress Reduction (MBSR) on sleep, mood, stress and fatigue symptoms in cancer outpatients. International Journal of Behavioral Medicine. 2005; 12(4):278-85. [DOI:10.1207/s15327558ijbm1204_9]

[57] Liu QQ, Zhou ZK, Yang XJ, Kong FC, Sun XJ, Fan CY. Mindfulness and sleep quality in adolescents: Analysis of rumination as a mediator and self-control as a moderator. Personality and Individual Differences. 2018; 122:171-6. [DOI:10.1016/j. paid.2017.10.031]

[58] Park M, Zhang Y, Price LL, Bannuru RR, Wang Ch. Mindfulness is associated with sleep quality among patients with fibromyalgia. International Journal of Rheumatic Diseases. 2020; 23(3):294-301. [DOI:10.1111/1756-185X.13756]

[59] Rosenzweig S, Greeson JM, Reibel DK, Green JS, Jasser SA, Beasley D. Mindfulness-based stress reduction for chronic pain conditions: Variation in treatment outcomes and role of home meditation practice. Journal of Psychosomatic Research. 2010; 68(1):29-36. [DOI:10.1016/j.jpsychores.2009.03.010]

[60] Flugel Colle KF, Vincent A, Cha SS, Loehrer LL, Bauer BA, Wahner-Roedler DL. Measurement of quality of life and participant experience with the mindfulness-based stress reduction program. Complementary Therapies in Clinical Practice. 2010; 16(1):36-40. [DOI:10.1016/j.ctcp.2009.06.008]

[61] Morone NE, Greco CM, Weiner DK. Mindfulness meditation for the treatment of chronic low back pain in older adults: A randomized controlled pilot study. Pain. 2008; 134(3):310-9. [DOI:10.1016/j.pain.2007.04.038]

[62] Morone NE, Greco CM. Mind-body interventions for chronic pain in older adults: A structured review. Pain Medicine. 2007; 8(4):359-75. [DOI:10.1111/j.1526-4637.2007.00312.x]

[63] Alemi S, Malihialzackerini S, Abolmaali Alhoseini Kh, Khabiri M. [Comparison of the effectiveness of mindfulness training and massage-aromatherapy on rising psychological health of elderly women with chronic pain (Persian)]. Iranian Journal of Health Education and Health Promotion. 2019; 7(4):359-70. [DOI:10.29252/ ijhehp.7.4.359] 
This Page Intentionally Left Blank 